\title{
MAO-B Elevation in Mouse Brain Astrocytes Results in Parkinson's Pathology
}

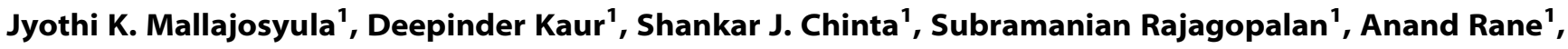 \\ David G. Nicholls', Donato A. Di Monte ${ }^{2}$, Heather Macarthur ${ }^{3}$, Julie K. Andersen ${ }^{1 *}$
}

1 Buck Institute for Age Research, Novato, California, United States of America, 2 Basic Science Research, Parkinson's Institute, Sunnyvale, California, United States of America, 3 Department of Pharmacological and Physiological Science, Saint Louis University School of Medicine, St. Louis, Missouri, United States of America

\begin{abstract}
Age-related increases in monoamine oxidase B (MAO-B) may contribute to neurodegeneration associated with Parkinson's disease (PD). The MAO-B inhibitor deprenyl, a long-standing antiparkinsonian therapy, is currently used clinically in concert with the dopamine precursor L-DOPA. Clinical studies suggesting that deprenyl treatment alone is not protective against PD associated mortality were targeted to symptomatic patients. However, dopamine loss is at least $60 \%$ by the time PD is symptomatically detectable, therefore lack of effect of MAO-B inhibition in these patients does not negate a role for MAO-B in pre-symptomatic dopaminergic loss. In order to directly evaluate the role of age-related elevations in astroglial MAO-B in the early initiation or progression of PD, we created genetically engineered transgenic mice in which MAO-B levels could be specifically induced within astroglia in adult animals. Elevated astrocytic MAO-B mimicking age related increase resulted in specific, selective and progressive loss of dopaminergic neurons in the substantia nigra (SN), the same subset of neurons primarily impacted in the human condition. This was accompanied by other PD-related alterations including selective decreases in mitochondrial complex I activity and increased mitochondrial oxidative stress. Along with a global astrogliosis, we observed local microglial activation within the SN. These pathologies correlated with decreased locomotor activity. Importantly, these events occurred even in the absence of the PD-inducing neurotoxin MPTP. Our data demonstrates that elevation of murine astrocytic MAO-B by itself can induce several phenotypes of PD, signifying that MAO-B could be directly involved in multiple aspects of disease neuropathology. Mechanistically this may involve increases in membrane permeant $\mathrm{H}_{2} \mathrm{O}_{2}$ which can oxidize dopamine within dopaminergic neurons to dopaminochrome which, via interaction with mitochondrial complex I, can result in increased mitochondrial superoxide. Our inducible astrocytic MAO-B transgenic provides a novel model for exploring pathways involved in initiation and progression of several key features associated with PD pathology and for therapeutic drug testing.
\end{abstract}

Citation: Mallajosyula JK, Kaur D, Chinta SJ, Rajagopalan S, Rane A, et al (2008) MAO-B Elevation in Mouse Brain Astrocytes Results in Parkinson's Pathology. PLoS ONE 3(2): e1616. doi:10.1371/journal.pone.0001616

Editor: Ashley Bush, Mental Health Research Institute of Victoria, Australia

Received August 22, 2007; Accepted January 28, 2008; Published February 20, 2008

Copyright: (C) 2008 Mallajosyula et al. This is an open-access article distributed under the terms of the Creative Commons Attribution License, which permits unrestricted use, distribution, and reproduction in any medium, provided the original author and source are credited.

Funding: These studies were funded via R01 NS40057-04 (JKA) and the National Parkinson's Foundation (JKM).

Competing Interests: The authors have declared that no competing interests exist.

*E-mail: jandersen@buckinstitute.org

\section{Introduction}

Monoamine oxidase $\mathrm{B}(\mathrm{MAO}-\mathrm{B})$ is found in the brain primarily in non-neuronal cells such as astrocytes and radial glia [1-3]. Its levels are known to increase with age and in association with neurodegenerative disease in both humans and mice [4-7]. Substrate oxidation by the enzyme is accompanied stoichiometrically by the reduction of oxygen to $\mathrm{H}_{2} \mathrm{O}_{2}[8,9]$. It has been postulated that age-related increases in MAO-B activity may contribute to cellular degeneration in the brain due to corresponding increases in the production of this reactive oxygen species (ROS)[10]. Although MAO-B is expressed primarily in astrocytes and not directly within dopaminergic cells, $\mathrm{H}_{2} \mathrm{O}_{2}$ has a high membrane permeability and therefore it can induce toxic effects not only within the cell of origin, but also in neighboring cells [11]. The area of the brain preferentially impacted in Parkinson's disease (PD), the substantia nigra (SN), contains high levels of MAO-B positive astrocytes which are themselves somewhat protected against the effects of $\mathrm{H}_{2} \mathrm{O}_{2}$ due to the fact that they contain high levels of both GSH and glutathione peroxidase which act in concert to detoxify $\mathrm{H}_{2} \mathrm{O}_{2}$ within cells [1115]. Neurons, which contain significantly lower levels of these protective components, are particularly vulnerable to this mild oxidizing agent [16-18]. This suggests that $\mathrm{H}_{2} \mathrm{O}_{2}$ produced within astrocytes by MAO-B may be either broken down to $\mathrm{H}_{2} \mathrm{O}$ within these cells or may diffuse to vulnerable nearby cells such as dopaminergic neurons [19]. MAO-B activity levels have been found to be doubled in the SN in Parkinson's disease, and to correlate with the percentage of dopaminergic SN cell loss [20]. This suggests that increases in MAO-B levels could play a role in subsequent dopaminergic cell death.

MAO-B-catalyzed ROS production has been suggested to contribute to an age-related increase in mitochondrial damage particularly in the SN [21]. Previous in vitro studies from our laboratory demonstrated that increases in $\mathrm{H}_{2} \mathrm{O}_{2}$ generation as a consequence of inducible increases in MAO-B levels results in selective inhibition of mitochondrial complex I activity which impacted on mitochondrial function within cultured dopaminergic cells [22]. Selective reductions in complex I activity are associated with $\mathrm{PD}$ and selective inhibition of complex I via systemic 
administration of either rotenone or 1-methyl-4-phenyl-1,2,3,6tetrahydropyridine (MPTP) results in patterns of morphological damage in rodents similar to that observed in the human Parkinsonian midbrain[23,24]. MAO-B is responsible for conversion of MPTP to $\mathrm{MPP}^{+}$within astrocytes from where $\mathrm{MPP}^{+}$can diffuse extracellularly. From there, it can be selectively transported into dopaminergic neurons via the dopamine transporter and elicit inhibitory effects on mitochondrial complex I activity [25].

There are numerous reports demonstrating the neuroprotective effects of the MAO-B inhibitors deprenyl or selegiline in Parkinsonian animal models although in many cases neuroprotection has been attributed to either antioxidant or antiapoptotic properties of the parent compounds or their metabolites [26-28]. Several multi-center studies including DATATOP, addressing the efficacy of selegiline administration in Parkinson's disease (PD) patients have concluded that selegiline both delayed and reduced the requirement of L-DOPA supplementation in early stages of symptomatic PD [29-31] but had no effect on the mortality rate [32]. Although deprenyl was found to be ineffective in slowing or halting the ultimate mortality in clinical PD it was given to patients only after symptoms were present which is known to coincide with the presence of an already extensive $(\sim 60 \%)$ midbrain dopaminergic cell loss [33]. These studies suggest that inhibition of MAO-B activity at this advanced stage in the disease is ineffective but this does not necessarily prove that MAO-B elevation is not a factor in initial neuropathology associated with the disorder and MAO-B inhibitors if given pre-symptomatically might not be protective. In order to test the hypothesis that elevations in MAO-B can directly contribute to pathologies observed in PD and to better understand the possible mechanisms underlying its effects on these parameters, we created genetically engineered mouse lines in which MAO-B levels can be inducibly increased specifically within astrocytes in adult animals. This allowed us to bypass any impact of its increased expression during the developmental time period. We report here that astrocytic elevation of MAO-B in adult mice recapitulates many of the pathological hallmarks associated with human PD and that these effects can be prevented by treatment with the selective MAOB inhibitor deprenyl or the antioxidant EUK189.

\section{Results}

\section{Creation of transgenic mouse lines in which astrocytic MAO-B activity can be inducibly increased in adult animals}

Double transgenic mouse lines were generated in our laboratory that constitutively express a reverse tetracycline responsive transactivator ( $\mathrm{rtTa}$ ) protein specifically within astrocytes via a glial fibrillary acidic protein promoter (pGFAP). Upon addition of the tetracycline derivative doxycycline (dox), rtTa binds to a tetracycline responsive bidirectional promoter (TET) and induces simultaneous expression of both wildtype human MAO-B cDNA (GenBank accession number NM 000898) and the marker protein bacterial beta-galactosidase (lacZ) (Figure 1A). Expression of the two co-transgenes was induced in our studies via inclusion of dox in the normal mouse chow, and visualized by RT-PCR (Figure 1B). Whole brain extracts from induced animals with the highest levels of expression exhibited an approximate 2.5-fold increase in MAO$\mathrm{B}$ and lacZ activities versus uninduced controls (Figure 1C); increased MAO-B activity was completely inhibited by deprenyl treatment (data not shown). This high-expressing line was used for all subsequent studies. Immunohistochemistry using antibodies specific to MAO-B demonstrated that the human MAO-B was exclusively expressed within (GFAP) astroglia throughout the brain and only following dox treatment (Figure 1D).

\section{Elevation in astrocytic MAO-B results in increased conversion of MPTP to MPP ${ }^{+}$and significant increases in toxin-induced dopaminergic SN cell loss}

To validate that our transgenic model expresses functionally active MAO-B, we assessed its vulnerability to acute systemic MPTP administration. Since conversion of MPTP to $\mathrm{MPP}^{+}$is catalyzed by MAO-B, we predicted that elevation in functional astrocytic MAO-B should result in increased striatal $\mathrm{MPP}^{+}$levels and exacerbation of selective MPTP-induced dopaminergic SN neurodegeneration. Induced and uninduced transgenic mice and wildtype C57Bl6 littermates were treated with $30 \mathrm{mg} / \mathrm{kg}$ MPTP and striatal $\mathrm{MPP}^{+}$levels measured via HPLC (Table 1). At 90 minutes post MPTP injection, striatal $\mathrm{MPP}^{+}$levels were 1.51.8-fold that observed in either uninduced transgenic or wildtype controls. This increase in striatal $\mathrm{MPP}^{+}$was accompanied by a significant decrease in striatal dopamine levels and dopaminergic SN cell numbers in induced transgenics compared to controls seven days following MPTP treatment (Figures 1E \& 1F). MAO-B elevation alone was found to result in $\sim 60 \%$ reduction in striatal dopamine levels (Figure 1E) which was exacerbated further by MPTP. Elevations in astrocytic MAO-B in our transgenics resulted in exacerbation of MPTP-induced neurotoxicity suggesting that astrocytic MAO-B increases had the expected functional effect.

Astrocytic MAOB elevation in mesencephalic cultures isolated from inducible MAO-B lines was found to result in dopaminergic neurodegeneration. Mesencephalic cultures were prepared from day-14 embryos from transgenic mice and wildtype littermates. Mixed neuronal/glial mesencephalic cultures were grown on coated plates for 3 days and transgenic MAO-B activity induced in a portion of the transgenic cultures by addition of $40 \mu \mathrm{g} / \mathrm{ml}$ dox. Dox was added to wildtype cultures as a negative control. Twelve hours following dox addition, immunocytochemistry performed on dox-induced transgenic cultures revealed a significant loss in the number of tyrosine hydroxylase positive $\left(\mathrm{TH}^{+}\right)$cells compared to controls even in the absence of toxin addition that was attenuated by deprenyl co-treatment (Figure 2A). To verify that loss of TH immunostaining represents actual preferential loss of dopaminergic cells, we counted the number of cells within the cultures which take up the fluorescent dopamine analogue $\mathrm{ASP}^{+}$(4-(4-(dimethylamino)styryl)-N-methylpyridinium iodide, Molecular Probes) as a measure of cells with functional dopamine transporter activity. We found a similar percentage loss of $\mathrm{ASP}^{+}$cells $(\sim 80 \%, 44 \pm 22$ versus $244 \pm 68$ uninduced per field). This demonstrates that the loss in $\mathrm{TH}^{+}$cell numbers is not simply a consequence of selective loss of this particular marker. In addition to increased dopaminergic cell loss, the remaining dopaminergic neurons were observed to undergo significant loss of their neurite processes which became stunted and shrunken in size. Deprenyl cotreatment attenuated not only the extensive dopaminergic cell loss observed following induction of MAO-B within cultured astrocytes but also the observed morphological dopaminergic neurite alterations (Figure 2B).

\section{Elevations of astrocytic MAO-B in vivo results in selective dopaminergic SN cell loss}

To assess the impact of astrocytic MAO-B elevation on its own in vivo on the nigrostriatal system, stereological dopaminergic cell counts were performed on dox-fed transgenic mice versus controls. A two week induction period in 2-3 month old transgenics resulted in an approximate $40 \%$ loss in dopaminergic SN cell numbers compared to either untreated transgenics or wildtype littermates; this loss was prevented by deprenyl co-treatment (Figure 3A). Moreover, removal of dox from the animal feed after 
A

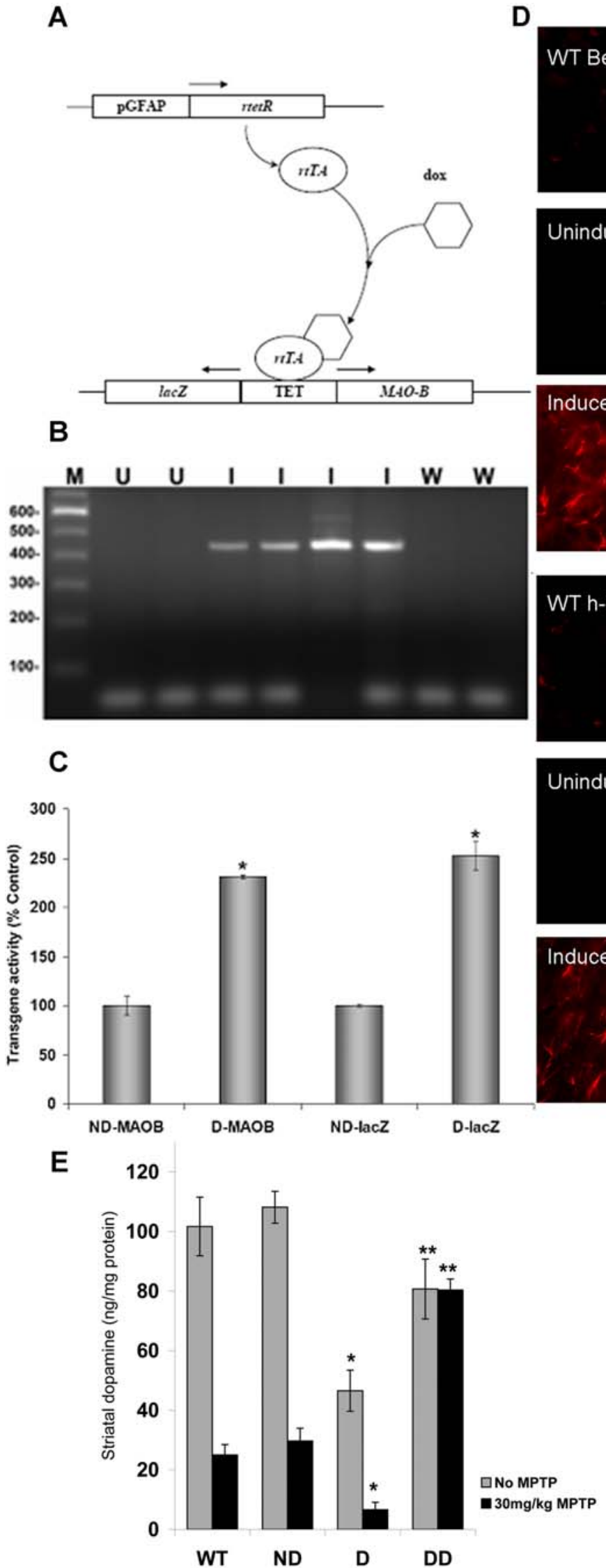

$\mathbf{F}$

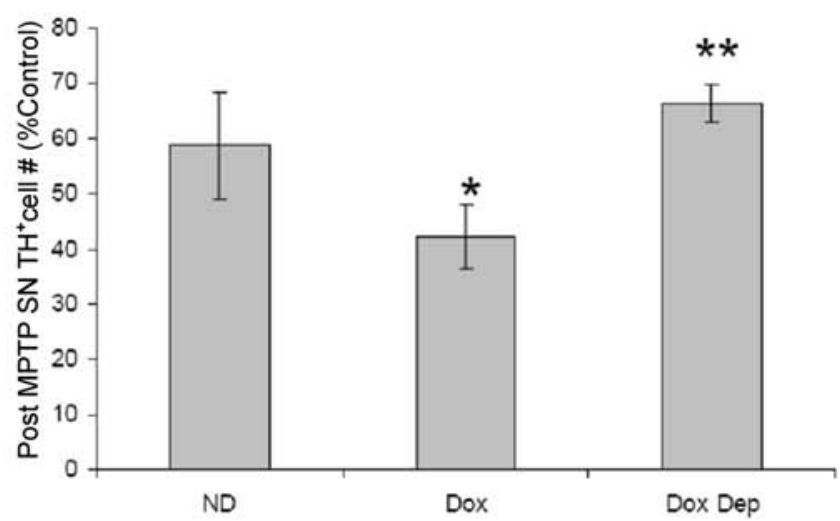


Figure 1. Characterization of transgenic mice that inducibly express human MAO-B specifically within astrocytes. A. Schematic of transgenic constructs utilized to create transgenic mice with inducible elevation of human MAO-B (h-MAO-B) expression specifically within astrocytes via the bacterial tetracycline regulatory system. A bacterial reverse tetracycline response gene (rtetR) was expressed constitutively via a mouse glial fibrillary acidic protein promoter (pGFAP). The resulting gene product, rtTA, a tet-responsive transactivator protein, is activated only in the presence of doxycycline (dox) and is able to bind to a tetracycline operator (TET) sequence in the promoter of a second transgene, resulting in induction of expression of both $\mathrm{h}-M A O-B$ and bacterial beta-galactosidase (lac Z) from the bidirectional promoter. B. Human MAO-B cDNA transgene is expressed in the brains of transgenic mice following dox induction. Inducible transgene mRNA expression (a 448 bp amplicon) was visualized by RT-PCR from RNA isolated from whole brains of uninduced (U), dox-induced (I) transgenic mice and wild type littermates (W). C. Enzymatic activities of h-MAO-B and lacZ transgenes in whole brain lysates from uninduced (ND) and doxinduced (D) transgenic animals. $n=3$ animals per condition run in triplicate; ${ }^{*} p<0.01$. Data is presented as percent ND control. ND lacZ activity was calculated to be $11.9 \pm 0.54$ nmoles ONPG converted/mg protein/hour and wild type C57BL6 lacZ activity (not shown) to be $11.3 \pm 0.13 \mathrm{nmoles}$ ONPG converted/mg protein/hour respectively. Brain MAO-B activity in tissue isolated from the uninduced transgenic (ND) was calculated to be $14.6 \pm 0.9 \mathrm{nmoles} \beta$-PEA converted/mg protein/hour and in tissues from wild type C57BL6 mice (not shown) to be $14.2 \pm 0.23 \mathrm{nmoles} \beta$-PEA converted/mg protein/hour. D. Dox-inducible expression of human MAO-B (h-MAO-B) and lacZ is localized within GFAP ${ }^{+}$astrocytes. GFAP immunostaining is shown in green, h-MAO-B is red, and merged in yellow in uninduced (No DOX) and dox-induced (DOX) animals. Scale bar applies to all images. E. Loss in striatal dopamine (ST DA) content is exacerbated in dox-induced transgenics following a single MPTP injection of $30 \mathbf{~ m g / k g}$ body weight. Striatal dopamine was estimated from wild type (W), uninduced transgenics (ND), induced transgenics (D), and dox-induced transgenics co-treated with deprenyl (Ddep). Grey bars without MPTP, black bars after a single injection of $30 \mathrm{mg} / \mathrm{kg}$ MPTP. Data is expressed as ng dopamine/mg striatal protein. ${ }^{*} p<0.01 \mathrm{D}$ vs. ND, ${ }^{* *}=p<0.01$ Ddep vs. ND. F. Loss in dopaminergic tyrosine-hydroxylasepositive $\left(\mathrm{TH}^{+}\right)$neurons in the substantia nigra (SN) after a single injection of MPTP $(30 \mathrm{mg} / \mathrm{kg})$ is exacerbated in dox-induced mice. Uninduced transgenic mice (ND), induced transgenics (Dox), and induced transgenics co-treated with 10mg/kg deprenyl (Doxdep). Data is presented as percent of non-MPTP treated ND controls where SN TH${ }^{+}$cell numbers are estimated at 13,318 $\pm 475 \mathrm{TH}^{+}$neurons in untreated ND mice (WT values, not shown, were $13,699 \pm 325) . n=3$ animals per condition run in triplicate; ${ }^{*} \mathrm{p}<0.05$.

doi:10.1371/journal.pone.0001616.g001

the initial two weeks for three weeks did not result in a reversal of $\mathrm{TH}^{+}$counts to normal levels. Astrocytic MAO-B elevation in older (14 mo.) transgenics resulted in an even more pronounced dopaminergic SN cell loss $(\sim 50 \%)$ than that observed in the younger animals. In contrast, total neuronal numbers in the $\mathrm{SN}$ as assessed by NeuN staining did not differ between dox-treated transgenics versus untreated controls nor were neuronal cell numbers in the striatum or cortex impacted by astrocytic MAO-B elevation (Figures 3B), even though it occurred throughout the brain and resulted in a general and widespread astrogliosis as evidenced by GFAP immunocytochemistry in these same brain regions (Figures 3C, D). GABAergic SN cell counts also revealed no difference in dox-induced versus non-induced animals further suggesting that the observed dopaminergic cell loss in this brain region was selective (data not shown). GFAP immunostaining revealed profuse branching as normally observed following astroglial activation and increased astroglial brain numbers following dox treatment which were inhibited by deprenyl. Levels of S100- $\beta$, a marker for activated astrocytes, were also increased in transgenic astroglia following dox induction (Figure 3E).

We performed silver staining to verify that selective loss of $\mathrm{TH}^{+}$ immunostained cells in the SN following dox induction coincided with increased neurodegeneration in this brain region (Figure 3F).

Table 1. Striatal MPP ${ }^{+}$levels.

\begin{tabular}{ll}
\hline MPP $^{+}$levels \\
\hline \\
\hline MPP \\
\hline Dox & (ng/mg protein) \\
No dox & $182 \pm 3.4$ \\
WT & $118 \pm 2.3$ \\
\hline
\end{tabular}

$\mathrm{MPP}^{+}$levels were measured in striatal tissues collected from wildtype (WT), uninduced (No dox) and dox-induced (Dox) transgenic mice 90 min post-MPTP injection $(30 \mathrm{mg} / \mathrm{kg})$ via HPLC with electrochemical detection. MPP ${ }^{+}$was extracted from tissues homogenized in the presence of $0.4 \mathrm{~N}$ perchloric acid and measured against a known standard. Values are presented as $\mathrm{ng} \mathrm{MPP}^{+} / \mathrm{mg}$ protein , $\mathrm{n}=3$ per treatment condition.

doi:10.1371/journal.pone.0001616.t001
This data along with the dox removal results suggests that the decrease in $\mathrm{TH}^{+} \mathrm{SN}$ cells is not merely due to a reversible selective loss of the $\mathrm{TH}^{+}$marker but constitutes an irreversible dopaminergic SN neurodegeneration.

\section{Astrocytic MAO-B elevations result in increased mitochondrial oxidative stress in dopaminergic SN neurons}

Based on our data, global increases in astrocytic MAO-B appeared to result in a preferential neurodegeneration of dopaminergic neurons suggesting that dopamine itself may be involved in the neurodegenerative process. Recent work in isolated brain mitochondria [34] demonstrated that dopamine oxidized to dopaminochrome (DACHR) can remove electrons from the autooxidizable site in mitochondrial complex I forming dopaminochrome radical. Dopaminochrome radical can in turn react with $\mathrm{O}_{2}$ present in the mitochondria converting it to $\mathrm{O}_{2}{ }^{-}$in concert with rereduction of the dopaminergic radical, setting up a redox cycling event. This would be manifest as an increase in mitochondrial $\mathrm{O}_{2}{ }^{-}$ levels and ROS-induced complex I inhibition within the dopaminergic neurons which could result in their selective demise. We hypothesized that membrane-permeant $\mathrm{H}_{2} \mathrm{O}_{2}$ produced by increased MAO-B activity within astrocytes could interact with intercellular dopamine and produce selective degenerative effects on dopaminergic neurons via a similar mechanism. To first test for the ability of astrocytes with elevated MAO-B activity to produce increased levels of extracellular ROS, we measured $\mathrm{H}_{2} \mathrm{O}_{2}$ generated in the culture medium in mesencephalic cultures isolated from our inducible MAO-B transgenics following dox induction. $\mathrm{H}_{2} \mathrm{O}_{2}$ levels released into the media in dox-induced cultures were significantly higher than those produced from untreated cultures (Figure 4). Cotreatment with deprenyl prevented the increase in extracellular $\mathrm{H}_{2} \mathrm{O}_{2}$ as did treatment with the antioxidant superoxide dismutasecatalase mimetic compound EUK-189. Co-treatment with apocynin, an NADPH oxidase inhibitor, resulted in a partial reduction in the $\mathrm{H}_{2} \mathrm{O}_{2}$ levels indicating that microglial activation contributes to the ROS increase although it is not clear whether this is directly due to astroglial activation or a secondary effect of dopaminergic cell death in the cultures. Ibal ${ }^{+}$cells $($a $17 \mathrm{kDa}$ calcium binding protein expressed in microglia) were found to constitute $3 \%$ of the total cell 
A

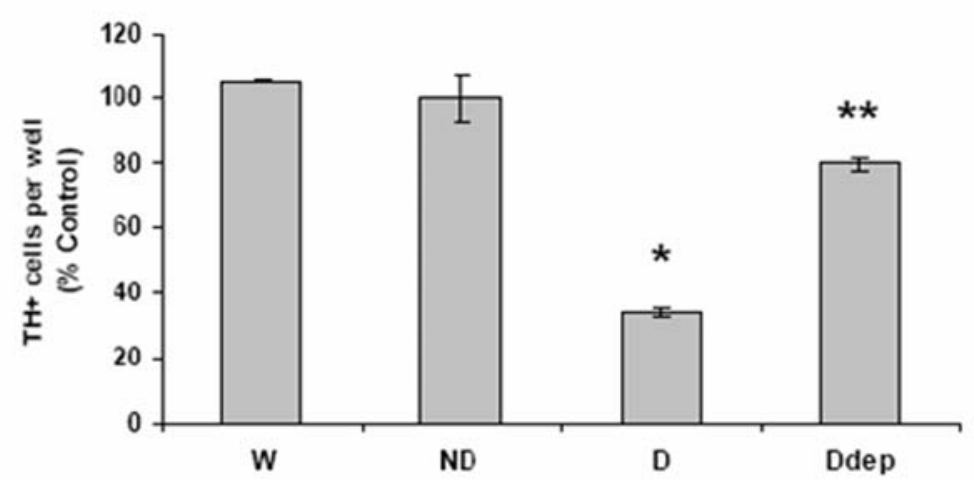

B

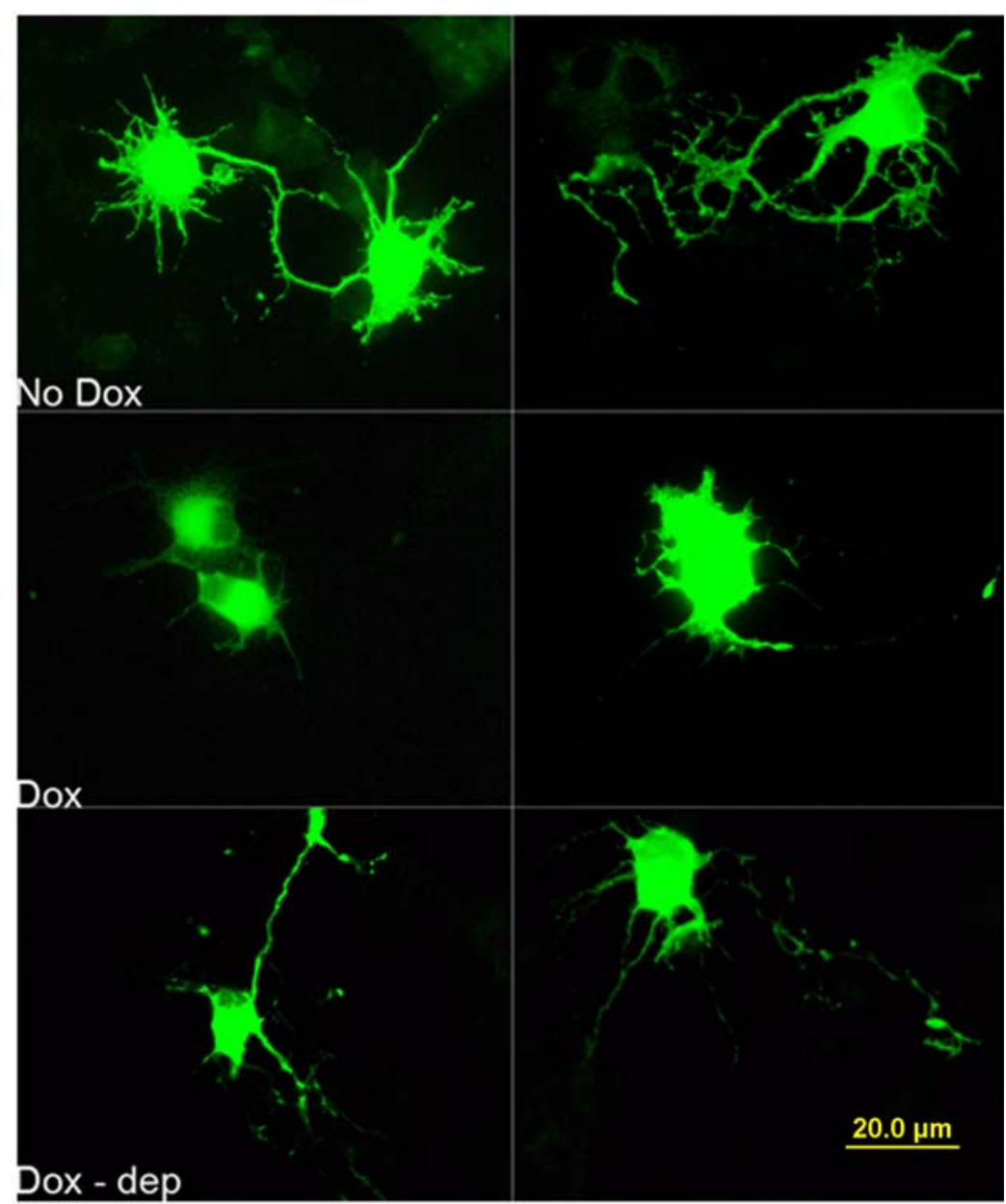

Figure 2. Inducible elevation of astrocytic MAO-B levels in mesencephalic cultures isolated from transgenic mice results in $\mathrm{TH}^{+}$cell loss which is prevented by co-treatment with deprenyl. A. $\mathrm{TH}^{+}$cell counts in uninduced mesencephalic cultures isolated from either wildtype littermates (WT), uninduced transgenics (ND) or transgenic cultures induced with $40 \mu \mathrm{g} / \mathrm{ml}$ doxycycline for $24 \mathrm{hrs}$ in the absence (D) or presence of $\mathbf{1 0}^{-6} \mathbf{M}$ deprenyl (Ddep). Counts are expressed as \% WT values which were estimated to be $300 \pm 15 \mathrm{TH}^{+}$neurons. Cell counts were performed in a minimum of three different wells and 4 separate fields in each well. ${ }^{*}=p<0.01$ compared to ND; ${ }^{* *} p>0.01$ compared to Dox. B. Representative micrographs of the $\mathbf{T H}^{+}$cells obtained by $\mathbf{T H}^{+}$immunofluorescence in uninduced (No Dox), dox-induced (Dox) and dox-induced mesencephalic cultures treated with deprenyl (Doxdep). Note the truncated processes and shrunken dopaminergic cell morphology in the induced cultures versus uninduced and deprenyl-treated induced cultures. doi:10.1371/journal.pone.0001616.g002

population and their numbers were increased by $\sim 17 \%$ following dox induction (data not shown).

Dopaminergic neurons constitute only a minor portion $(\sim 5 \%)$ of cells making up the $\mathrm{SN}$ (unpublished observations). In contrast to the $\mathrm{SN}$, the striatum (ST) is larger, easier to dissect out, and at least $10-15 \%$ of the striatal nerve terminals originate from SN dopaminergic neurons [35-37]. In order to achieve meaningful data as to the impact of astrocytic MAO-B elevation on $\mathrm{H}_{2} \mathrm{O}_{2}$ levels and mitochondrial function selectively within dopaminergic nigrostriatal neurons, we chose to enrich for striatal dopaminergic 


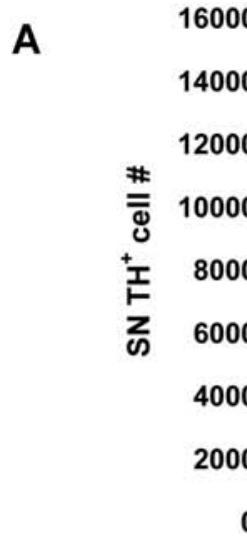

B

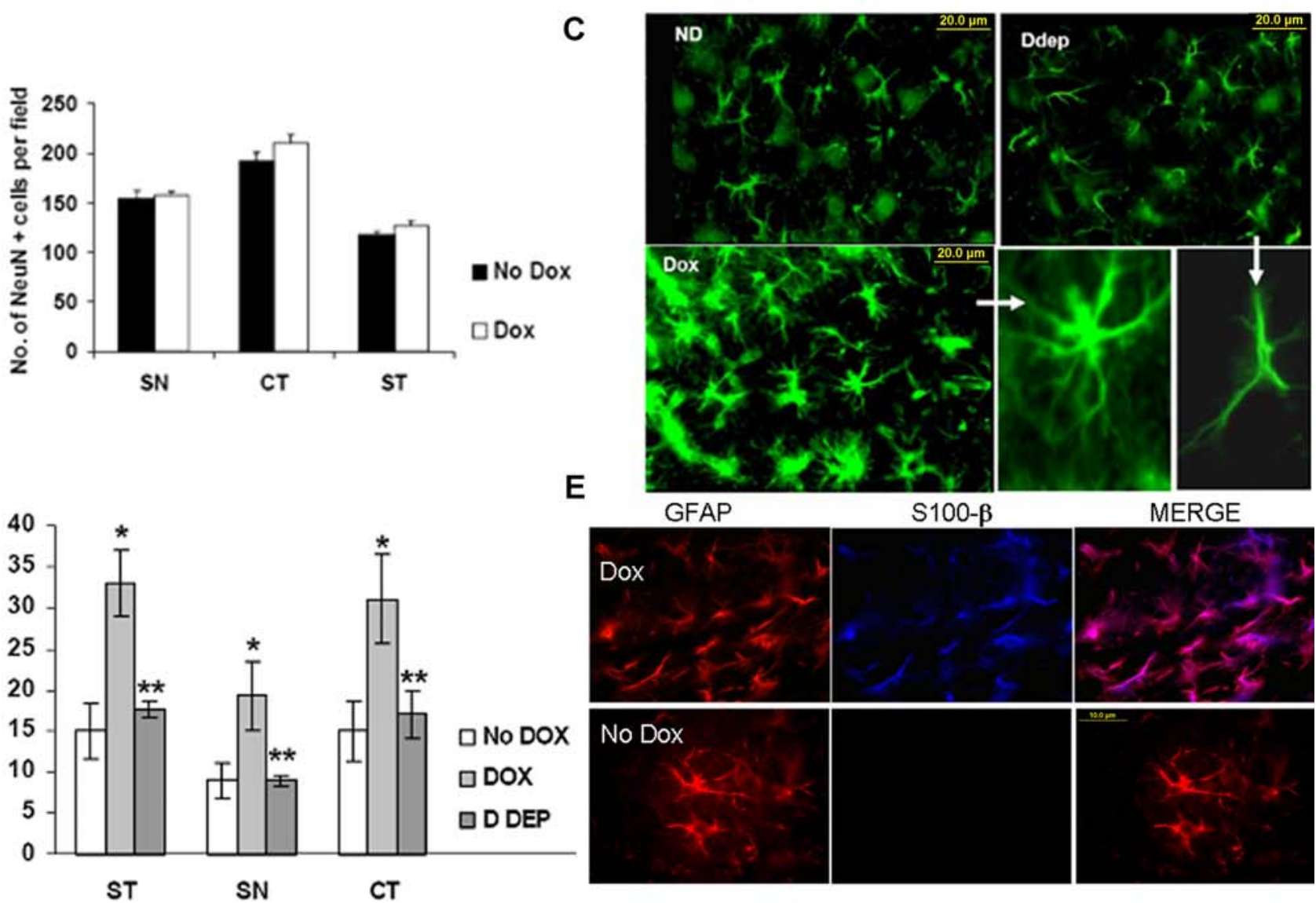

$\mathbf{F}$

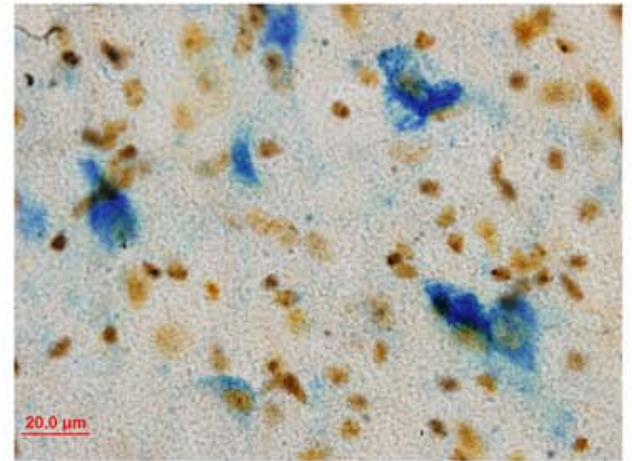

No Dox

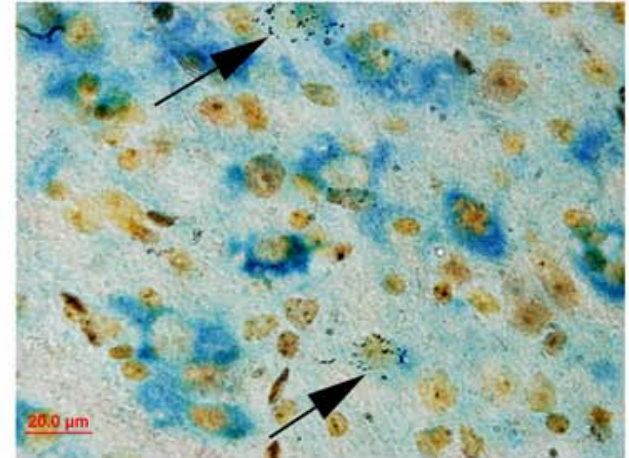

Dox 
Figure 3. Inducible elevation in astrocytic MAO-B results in selective, age-related SN dopaminergic neurodegeneration and global astrocyte activation even in the absence of neurotoxin. $\mathrm{A}^{\text {. }} \mathrm{TH}^{+} \mathrm{SN}$ cell counts from uninduced transgenics (no dox) versus induced transgenics at 3-4 months (dox), following dox removal (dox rem) at 14 months of age (dox 14 mo), and induced co-treated with deprenyl at 10-30 mg/kg (dox dep 10-30), the anti-inflammatory agent minocycline at $10 \mathrm{mg} / \mathrm{kg}$ (dox mino) or the anti-oxidant Euk 189 at $10 \mathrm{mg} / \mathbf{k g}$ in young animals. Data is expressed as total number of $S N \mathrm{TH}^{+}$cells numbers per animal; * $\mathrm{p}<0.01$ compared to ND, ${ }^{* *} \mathrm{p}<$ 0.05 compared to Dox, and *** $p<0.05$ compared to dox. B. Neuronal (NeuN ${ }^{+}$) counts from uninduced (No Dox, filled bars) versus doxinduced transgenics (Dox, open bars) performed in equivalent nigral (SN), cortical (CT), and striatal (ST) tissue sections. Values represent average cell numbers per field in three separate fields per section, three sections per animal. $\mathrm{N}=5$ animals per group. C. Representative micrographs demonstrating cortical astrocytic activation in dox-induced (Dox) versus uninduced (ND) animals and dox-induced animals co-treated with deprenyl (Ddep). Astrocytes from induced transgenics displayed enhanced GFAP staining and increased branching indicative of astrocyte activation (higher magnification in lower middle panel); deprenyl treatment resulted in reduced branching similar to that observed in uninduced controls (higher magnification in lower right-hand panel). D. Astrocytic (GFAP ${ }^{+}$) counts from uninduced (No DoX, open bars) versus dox-induced transgenics (DOX, light grey bars) and mice dox-induced in the presence of deprenyl co-treatment (D DEP, dark grey bars) performed in equivalent striatal (ST), nigral (SN), and cortical (CT) tissue sections. Values represent average cell numbers per field at $40 \times$ magnification in three separate fields per section, three sections per animal. $N=5$ animals per group; ${ }^{*} \mathrm{p}>0.05$, ${ }^{* *} \mathrm{p}<0.1$. $\mathbf{E}$. Representative micrograph demonstrating increased expression of the astrocytic activation marker S-100 $\beta$ (blue) in GFAP ${ }^{+}$cells in the transgenic cortex following dox induction. GFAP-labeled astrocytes (red) in induced animals display increased S-100 $\beta$ staining (pink) versus uninduced. F. Neurodegeneration of $\mathbf{T H}^{+}$cells as assessed by silver staining. SN sections from uninduced (No Dox) and induced (Dox) animals were processed for $\mathrm{TH}^{+}$immunoreactivity visualized by alkaline peroxidase (blue) and further processed for silver staining. A black punctate staining was observed in and around dying $\mathrm{TH}^{+}$neurons (arrows) in Dox but not No Dox fields.

doi:10.1371/journal.pone.0001616.g003

nerve terminal (synaptosomal) populations using antibody against DAT via a modified immunomagnetic bead approach (Fig. 5A,B). Synaptosomes in the flow-through fractions were non-dopaminergic $\left(\mathrm{GABA}^{+}\right)$and therefore used as negative controls in our subsequent biochemical analyses.

$\mathrm{H}_{2} \mathrm{O}_{2}$ levels were measured in isolated ST dopaminergic synaptosomes following injection of DCF into the tail vein of dox-induced versus uninduced and wildtype littermate controls as modified from Andrews et al. [38]. $\mathrm{H}_{2} \mathrm{O}_{2}$ levels was found to be elevated in isolated ST dopaminergic synaptosomes following increased astrocytic MAO-B elevation; this was found to be preventable by co-treatment of animals with either deprenyl or EUK-189 (Fig. 5C). Catalase treatment of the dopaminergic synaptosomes from the induced animals reduced the fluorescence to below uninduced levels indicating that the DCF fluorescence is due to $\mathrm{H}_{2} \mathrm{O}_{2}$. In conjunction with elevations in $\mathrm{H}_{2} \mathrm{O}_{2}$ within nerve terminals originating from dopaminergic $\mathrm{SN}$ neurons, we found an elevation in striatal DACHR. DACHR is a stable derivative of dopamine quinone, the major species produced by dopamine oxidation [39] (Figure 5D). Along with increases in DACHR levels, we found a preferential reduction in rotenoneinhibitable complex I (CI) activity in the ST dopaminergic versus non-dopaminergic synaptosomes (Figure 5E). In contrast, no change in complex IV activity was observed in either population following dox-induction (Figure 5E). Loss of CI activity was attenuated in dopaminergic synaptosomes isolated from doxinduced animals that had been co-treated with deprenyl or EUK-189.

To assess whether increased dopaminergic $\mathrm{H}_{2} \mathrm{O}_{2}$ and DACHR levels following astrocytic MAO-B elevation in vivo resulted in corresponding increases in mitochondrial $\mathrm{O}_{2}{ }^{-}$levels within dopaminergic cells as previously demonstrated in vitro by Zoccarato and colleagues [34], animals were administered the superoxide indicator mitosox red via tail vein injection[38]. Doxinduced transgenics displayed elevated presence of mitosox red staining within mitochondria in both $\mathrm{TH}^{+}$fibers in the striatum and in $\mathrm{TH}^{+}$neurons within the $\mathrm{SN}$ versus uninduced controls (Figure 6, Table 2). Increases in mitosox red fluorescence within the dopaminergic SN neurons were prevented by co-treatment with either deprenyl or EUK189 (Figure 6, Table 2). Significantly, dopaminergic SN cell loss was found to be attenuated in the presence of co-treatment with not only deprenyl, but also EUK189 suggesting that oxidative stress is an important component of dopaminergic demise (Figure 3A).

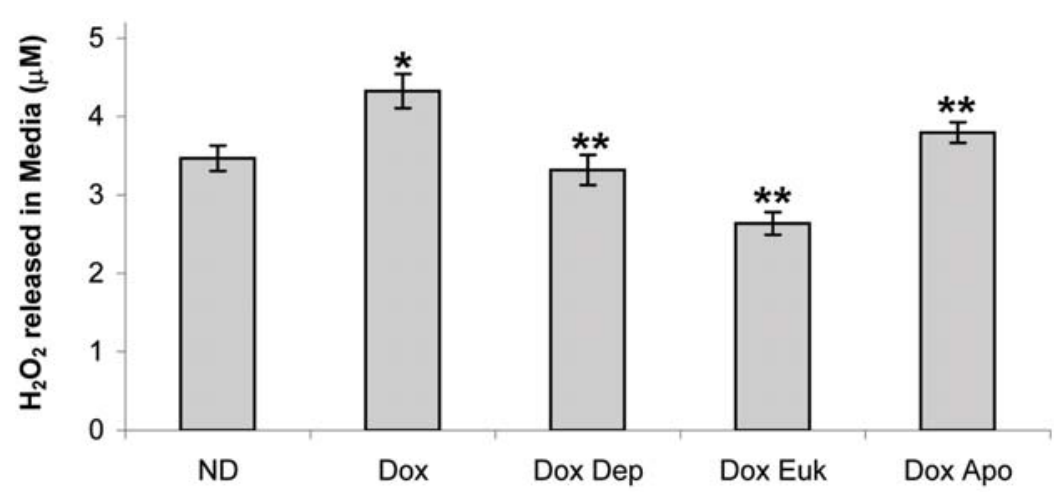

Figure 4. Elevation in astrocytic MAO-B results in increased extracellular hydrogen peroxide $\left(\mathbf{H}_{\mathbf{2}} \mathrm{O}_{\mathbf{2}}\right)$. Extracellular $\mathrm{H}_{2} \mathrm{O}_{2}$ concentrations were measured in media isolated from uninduced (ND) versus dox-induced (Dox) mesencephalic cultures in the absence and presence of $10 \mu \mathrm{M}$ deprenyl (Dox Dep), $30 \mu$ M EUK189 (Dox Euk) or $500 \mathrm{nM}$ apocynin (Dox Apo) using the Amplex Red assay. $\mathrm{H}_{2} \mathrm{O}_{2}$ concentrations were quantified against a standard $\mathrm{H}_{2} \mathrm{O}_{2}$ curve. Measurements were made on three separate media aliquots harvested prior to immunostaining of cultures; ${ }^{*} p<0.01$ compared to ND, ** $\mathrm{p}<0.05$ compared to ND.

doi:10.1371/journal.pone.0001616.g004 
A

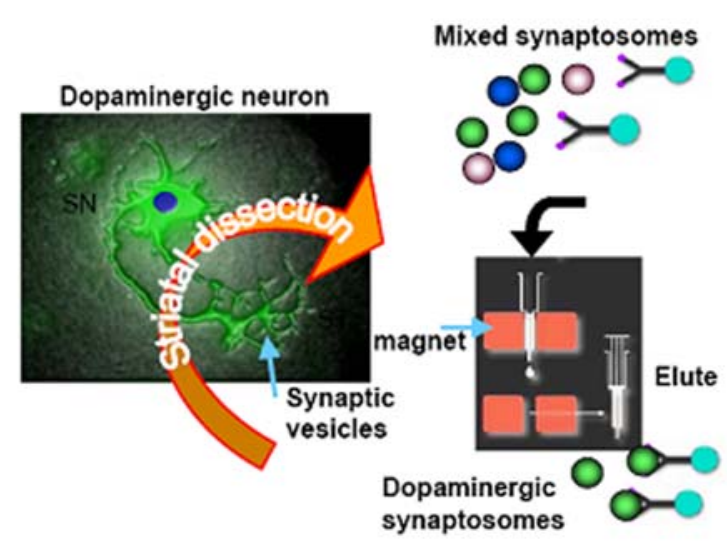

IMMUNO-MAGNETIC ISOLATION OF DOPAMINERGIC SYNAPTOSOMES

C

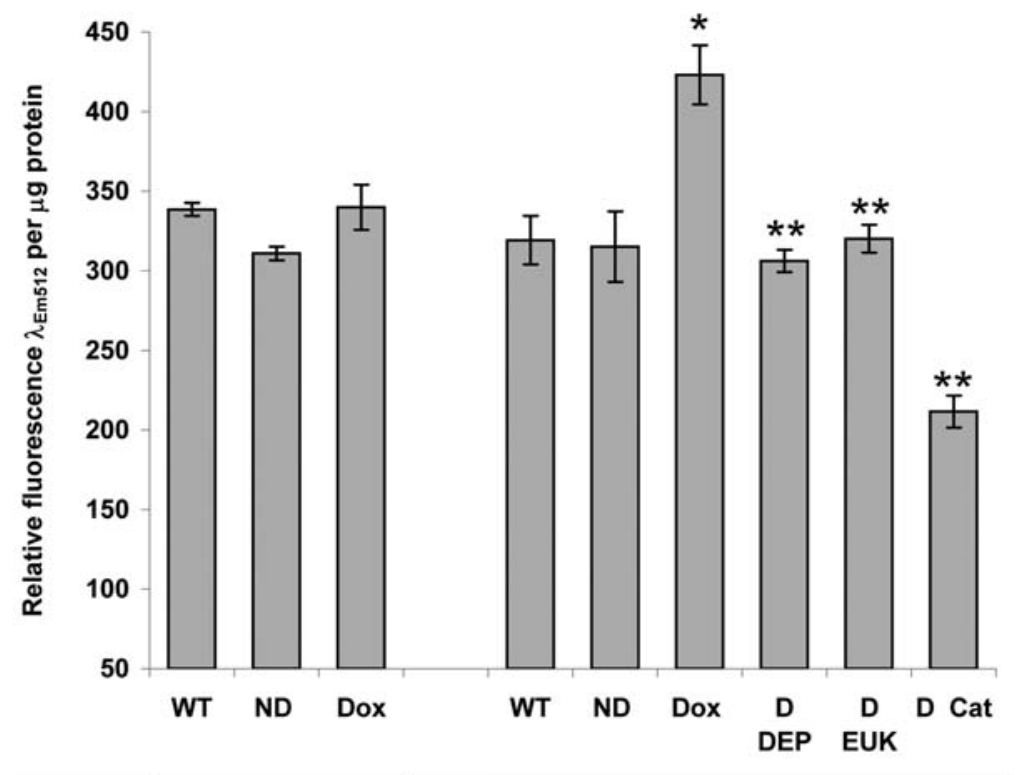

B

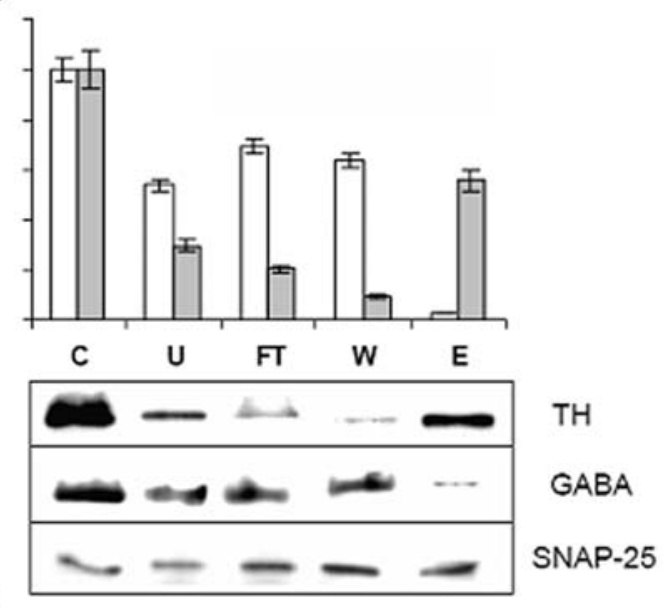

D
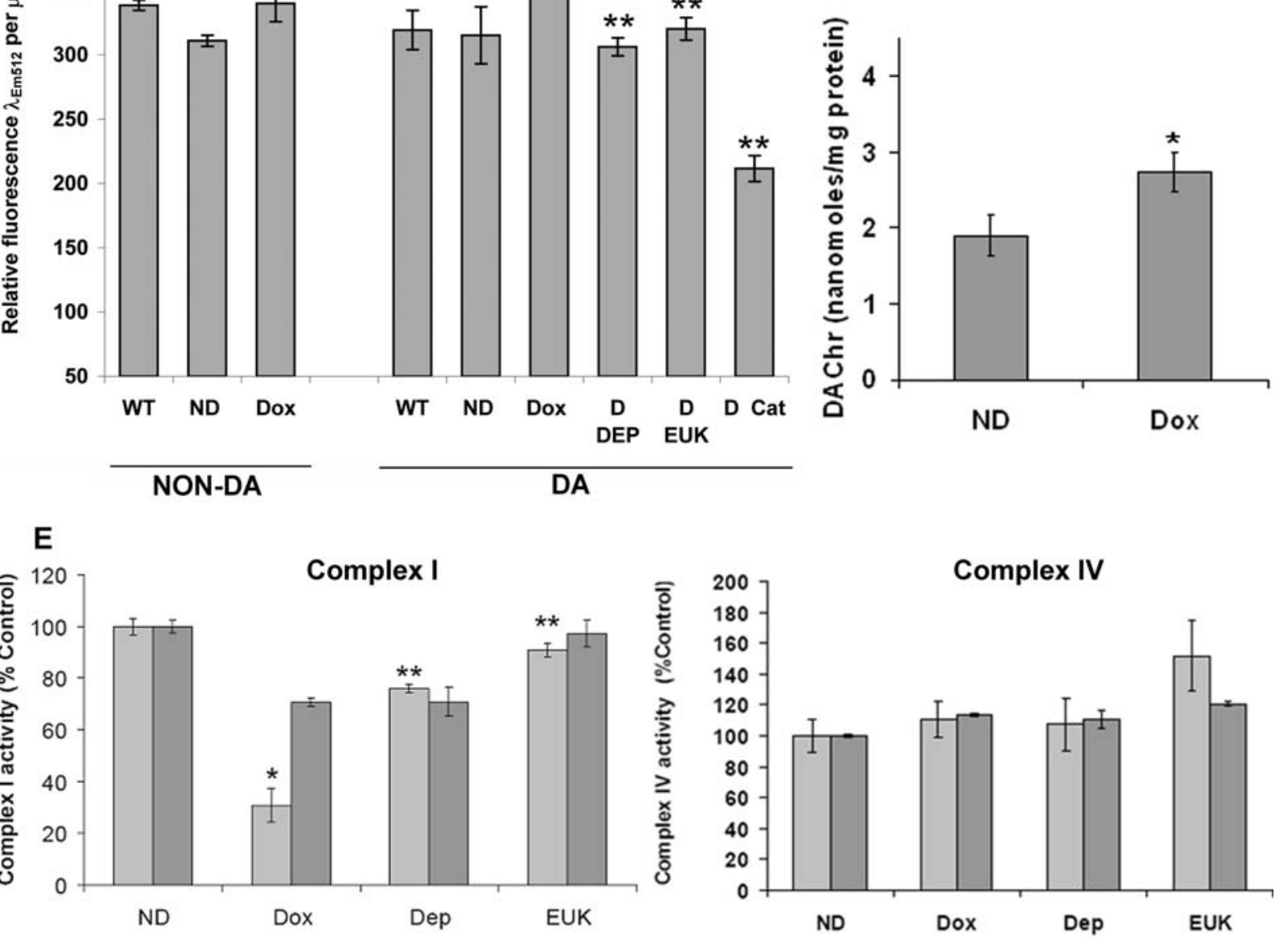

Figure 5. Effects of elevations of astrocytic MAO-B on $\mathrm{H}_{2} \mathrm{O}_{2}$ and dopaminochrome (DAChr) levels, and mitochondrial complex I versus IV activities in isolated ST dopaminergic versus non-dopaminergic synaptosomes. A. Schematic of novel immunomagnetic technique utilized to for isolate ST dopaminergic versus non-dopaminergic synaptosomes. Synaptosomes were prepared from freshly dissected striata and the dopaminergic population rapidly isolated via immunoprecipitation with anti-DAT antibody following by separation by a magnetic particle-conjugated secondary antibody running through a strong magnetic column. Elution of the DA synaptosomes was obtained via removal of the magnetic field. Flow-through contains striatal non-dopaminergic synaptosomes used as negative controls in subsequent biochemical experiments. B. Densitometric quantitation of western blot analyses of fractions from immunomagnetic isolation of ST 
synaptosomes probed with TH, GABA or SNAP-25 antibodies. Levels of Dopaminergic TH (grey bars) versus Non-dopaminergic GABA (white bars) synaptosomes were assessed using SNAP-25, a general synaptic protein, as a loading control. Control (C), unbound fraction (U), flow-through $(\mathrm{FT})$, wash (W) and eluate (E). Representative blots are shown in the lower panel. C. Elevation in astrocytic $\mathbf{M A O}-\mathbf{B}$ results in increased $\mathbf{H}_{\mathbf{2}} \mathbf{O}_{\mathbf{2}}$ within dopaminergic ST synaptosomes. $\mathrm{H}_{2} \mathrm{O}_{2}$ levels were estimated $3 \mathrm{hrs}$ following tail vein injection of DCFDA into either wildtype littermates (WT), uninduced (ND) or dox-induced (Dox) transgenics, or induced transgenic animals co-treated with either deprenyl (D DEP), EUK189 (D EUK) or $1 \mathrm{mg} / \mathrm{ml}$ catalase (Dox Cat). DCF fluorescence was examined at an excitation wavelength of $488 \mathrm{~nm}$ and emission at $512 \mathrm{~nm}$. Data is reported normalized to microgram synaptosomal protein. $N=3 ;{ }^{*} p<0.005$ versus $N D,{ }^{* *}=p<0.01$ versus Dox. D. Elevation in astrocytic MAO-B results in increased ST DAChr levels. DAChr levels were estimated in ST samples from uninduced (ND) versus dox-induced (Dox) transgenic mice via HPLC. Values are presented as picomoles DAChr/mg protein.* $p<0.02, n=3$. E. Elevation in astrocytic MAO-B results in decreased complex I but not complex IV activity in ST dopaminergic synaptosomes. Complex I and complex IV activities were measured in isolated ST dopaminergic versus non-dopaminergic synaptosomes from uninduced (ND), dox-induced (Dox) and induced transgenic animals co-treated with either deprenyl (Dep) or EUK-189 (EUK). N = 3-5 animals per group and a total of 3 separate experiments, values are expressed as mean \pm SD; * $p<0.01$ compared to $\mathrm{ND},{ }^{* *} \mathrm{p}>0.05$ compared to ND. Cl activity was calculated to be 4.0 versus $3.3 \mu \mathrm{M} \mathrm{NADH} / \mathrm{min} / \mathrm{mg}$ protein in ND striatal dopaminergic and nondopaminergic synaptosomes, respectively. CIV activity was calculated to be 139 versus $109 \mu \mathrm{M}$ ferrocytochrome c/minute/mg protein in ND striatal dopaminergic and non-dopaminergic synaptosomes, respectively. Activities of WT DA striatal synaptosomes was calculated to be $4.4 \mu \mathrm{M}$ NADH/min/ $\mathrm{mg}$ protein $(\mathrm{Cl})$ and $130 \mu \mathrm{M}$ ferrocytochrome $\mathrm{c} /$ minute/mg protein (CIV); dox feeding of the WT animals did not alter the activities. Note: addition of doxycycline has been reported to interfere with mitochondrial protein translation including $\mathrm{Cl}$ thereby affecting enzyme activity [62], however in addition to lack of effect on $\mathrm{Cl}$ activity in WT animals +/- dox, we also find no change in Cl subunit protein levels in WT mice as a consequence of dox treatment as assessed via immunoprecipitation/gel electrophoretic analyses (data not shown).

doi:10.1371/journal.pone.0001616.g005

Increases in astrocytic MAO-B activity results in increased local microglial activation in the SN

Data from our mitosox red studies not only suggested that SN dopaminergic neurons display an increase in oxidative stress following astrocytic MAO-B elevation, but also in the activated local SN microglial cells (Fig. 7A). This is likely a secondary effect subsequent to dopaminergic SN neurodegeneration triggering local microgliosis. Degeneration of SN dopaminergic neurons in PD has been observed to be accompanied by local microglial activation [40]. It has also been noted post-mortem in both humans and primates exposed to MPTP $[41,42]$. It is detected in the chronic mouse rotenone model prior to appearance of the
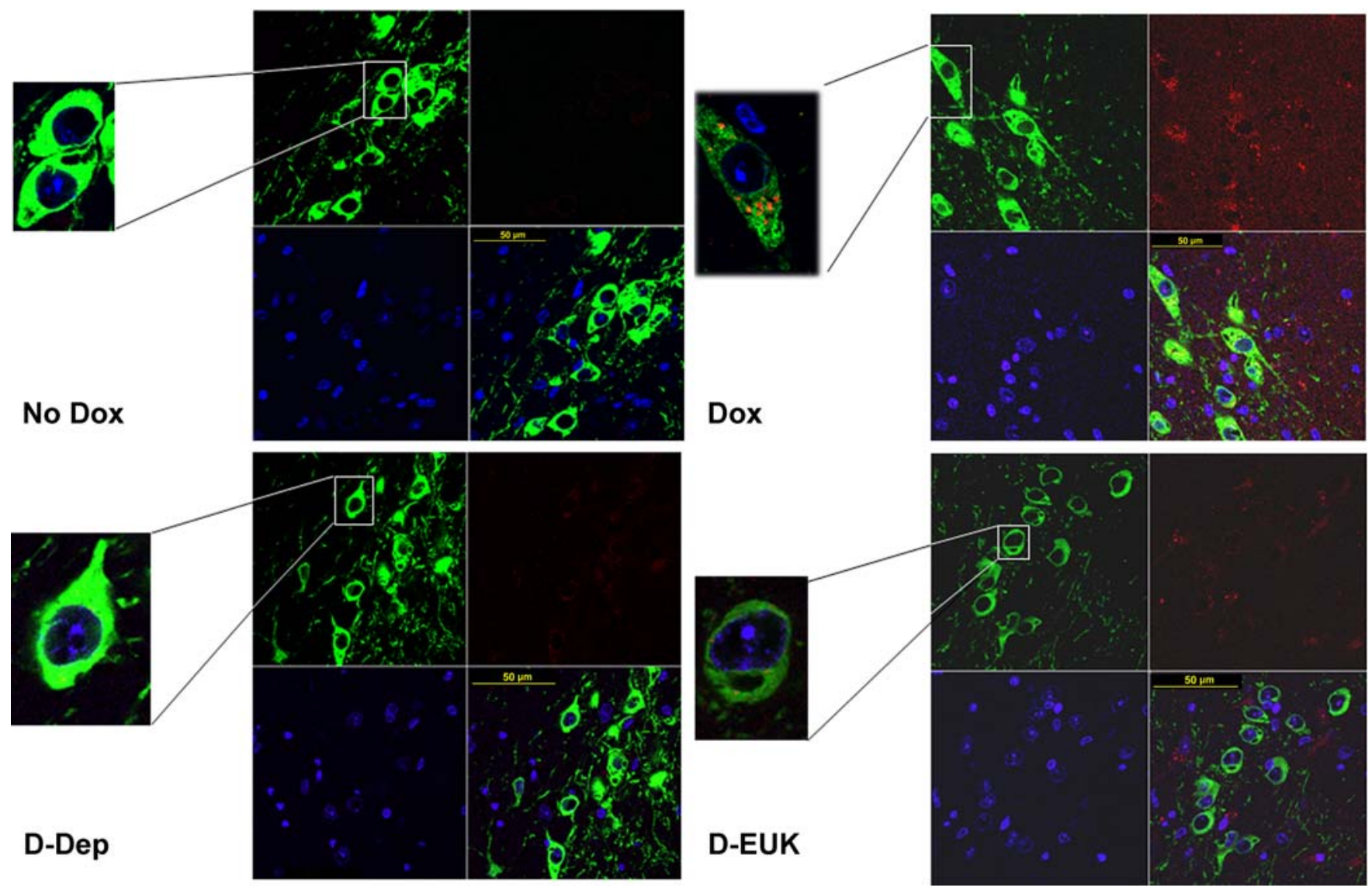

Figure 6. Elevation in astrocytic MAO-B results in increased mitochondrial superoxide levels within dopaminergic SN mitochondria. Representative confocal micrographs illustrating increased merged (yellow) mitosox red fluorescence (red) in the SN and striatum of dopaminergic neurons following tail vein injection, fixation and TH immunochemistry (green) in uninduced (No Dox) versus induced transgenic mice (Dox) which is prevented in the presence of co-deprenyl (DEP) or EUK-189 (EUK) treatment. Nuclei are visualized via DAPI staining (blue). doi:10.1371/journal.pone.0001616.g006 
Table 2. Superoxide in the SN dopaminergic neurons.

\begin{tabular}{llll}
\hline & Mean pixel intensity & & Total pixel fluorescence \\
\cline { 2 - 2 } & per cell & per cell \\
\hline ND & $10.8 \pm 2.0$ & $101,983 \pm 17,686$ \\
D & $24.2 \pm 3.6$ & $223,526 \pm 28,225$ \\
DD & $10.2 \pm 2.1$ & $91,937 \pm 19,107$ \\
DEUK & $15.4 \pm 4.0$ & $126,757 \pm 23,798$ \\
\hline
\end{tabular}

Confocal quantitation of relative mitosox-red fluorescence intensity in SN DA neurons in untreated (ND) or dox-treated (D) transgenic mice in the absence or presence of deprenyl (DD) or EUK-189 (DEUK). Confocal images from immunofluorescent sections of brains were obtained following various treatment regimes and subjected to intensity measurements using the IMARIS software suite (Bitplane AG, Zurich, Switzerland) on selected cells from multiple sections from each treatment. Data is presented both as mean pixel intensity and total red fluorescent pixels (mitosox red) per cell.

doi:10.1371/journal.pone.0001616.t002

dopaminergic lesion [43] and during selective SN dopaminergic neurodegeneration in the spontaneous weaver mouse mutant [44]. We performed immunochemistry to evaluate microglial activation in our induced MAO-B transgenics via Ibal immunostaining [45]. Prior to activation, microglia normally exhibit a highly ramified morphology. In response to an activating signal, microglia begin to withdraw their ramified branches and to extend new protrusions. Next they begin migration through the tissue, engulfing cells [46]. Astrocytic MAO-B expression was found to lead to local microglial activation in both the SN and the striatum (Figure $7 \mathrm{~B}-\mathrm{D}$ ) but not the cortex (supplement Figure S1). Unactivated ramified microglia were found in the uninduced nigrostriata and cortex; activated microglia with reduced branching in locomotory stages were only present within the $\mathrm{SN}$ and striatum of induced MAOB transgenics. In some cases, the microglia were in a high motile state with minimal processes or were in close contact with $\mathrm{TH}^{+}$neurons, possibly in the phagocytic stage (Figure 7C). Microglial activation was prevented by treatment with deprenyl (Figure 7B). Cotreatment of animals with an inhibitor of microglial activation, minocycline, prevented dopaminergic SN cell death suggesting that microglial activation plays a major role in dopaminergic neurodegeneration in this model (Figure 3A).

\section{Increases in astrocytic MAO-B activity results in decreased locomotor movement}

Finally, astrocytic increases in MAO-B in our model were found to correlate with a significant inhibition of locomotor function. Open field analysis of dox-treated mice revealed a significant difference in locomotor behavior in induced versus uninduced transgenics (Figure 8A, B). After treatment with dox alone for two weeks, induced mice displayed a $\sim 32-35 \%$ decrease in locomotor activity in comparison to uninduced littermates. In contrast, deprenyl-treated induced mice were completely unaffected.

\section{Discussion}

Increased brain MAO-B levels have been hypothesized to play a role in neuropathies associated with PD [47-51] however direct proof of a causative role has been thus far lacking. In this study, we demonstrate that elevations in astrocytic MAO-B levels results in a relatively selective loss of dopaminergic $\mathrm{SN}$ neurons and the severity of this loss appears to be age-dependent. It is not clear why there was not a more dramatic increase in SN DA cell loss with age in the MAO-B transgenics. Normally, cell loss coincides with an age-related increase in MAO-B enzyme activity. Our results suggest that perhaps the elevation of MAO-B to aging levels in the young animals was sufficient on its own to produce significant cell loss and that the additional stress of an aging brain contributed marginally (yet significantly) to this effect. Observed cell loss was accompanied by increased mitochondrial oxidative stress and selective decreases in mitochondrial complex I activity in these cells along with local microglial activation all of which we assume contribute to subsequent cell death. These pathological alterations were found to correlate with a significant decrease in locomotory behavior. Our data, taken in total, demonstrates that elevations in levels of astrocytic MAO-B activity results in several of key pathological hallmarks of Parkinson's disease.

ROS produced by MAO-B-expressing astrocytes can be released into the extracellular environment and, due to its high membrane permeability, diffuse into neighboring cells. Within dopaminergic neurons, it can oxidize dopamine to DACHR which can interact with electrons at the auto-oxidizable site of mitochondrial complex I at a higher affinity than oxygen itself producing DACR radical. Electrons can then be transferred from DACR radical to oxygen resulting in re-reduction of DACR to DACHR and production of superoxide as part of an ongoing redox cycling event. These events are shown schematically in Figure 9. We have demonstrated in this current study that increased astrocytic MAO-B results in elevations in extracellular ROS levels and increased intracellular $\mathrm{H}_{2} \mathrm{O}_{2}$ within the terminals of SN dopaminergic neurons accompanied by increased dopamine oxidation to DACHR, selective mitochondrial complex I inhibition and elevations in mitochondrial superoxide levels. These events are prevented by either co-treatment with the MAO-B inhibitor deprenyl or the antioxidant compound EUK189 which also prevented subsequent dopaminergic SN cell loss.

Another prominent feature noted in our model was local microglial activation within the nigrostriatum. Dramatic local proliferation of microglial cells has been reported in the postmortem PD brain as well as in various animal models of the disease $[40,43,44,52-54]$.Local microglial activation was found to be associated in our model with degenerating $\mathrm{TH}^{+}$cells in both the $\mathrm{SN}$ and the striatum. It may be a secondary reaction to initiation of dopaminergic neurodegeneration. Significantly, dopaminergic SN cell loss is substantially attenuated by co-treatment of animals with the microglial activation inhibitor minocycline suggesting that microglial activation plays a significant role in dopaminergic demise associated with this model.

Taking in total, our data demonstrates a linkage between several separate pathological events associated with Parkinson's disease that have not necessarily been considered to be mechanistically connected including mitochondrial dysfunction, and microglial activation. This suggests that MAO-B may be a common initiator for these events and provides a novel model for exploring the mechanisms by which these events can occur in the context of the human condition.

\section{Materials and Methods}

\section{Generation and analysis of transgenics with inducible astrocyte-specific elevation of MAO-B levels}

Human MAO-B cDNA was cloned into pBIG (Clontech) at the PstI and SalI sites, placing the gene under transcriptional control of the bidirectional Tet-inducible promoter. A second construct was prepared which contained the $3.7 \mathrm{~kb}$ HinDIII-BamHI fragment of the mouse GFAP promoter cloned in EcoRI blunt ended pTetOn vector, driving expression of the Tet-responsive reverse transactivator rtTA. Transgenic C57Bl6 mice inducibly expressing human MAO-B selectively within astrocytes were 
A
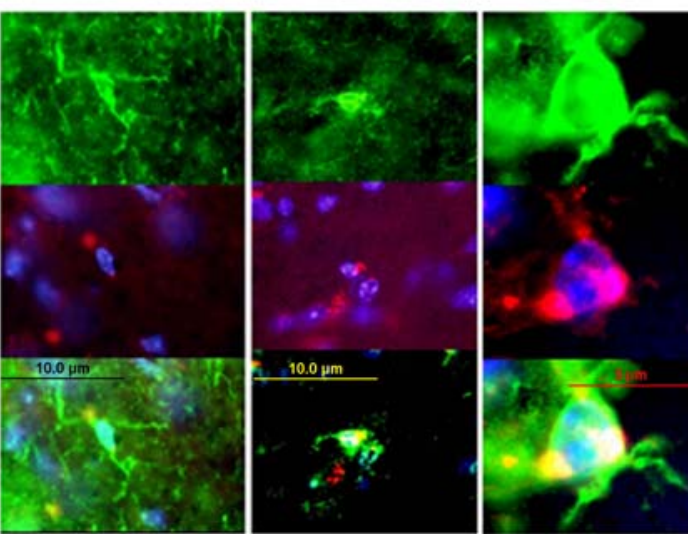

Iba1

No DOX-SN
Resting Microglia

B

Dox - SN

Detail

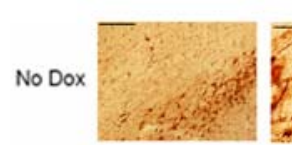

Activated Microglia

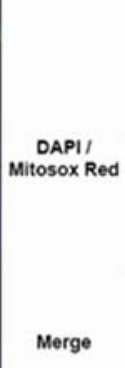

Dox
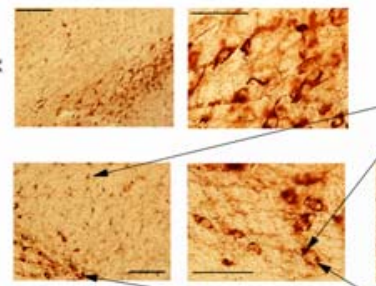

Iba1

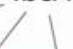

microglia

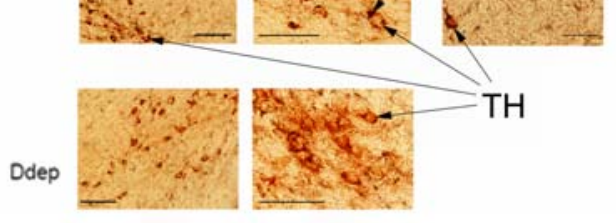

Activated

microglia

Few to no

microglia

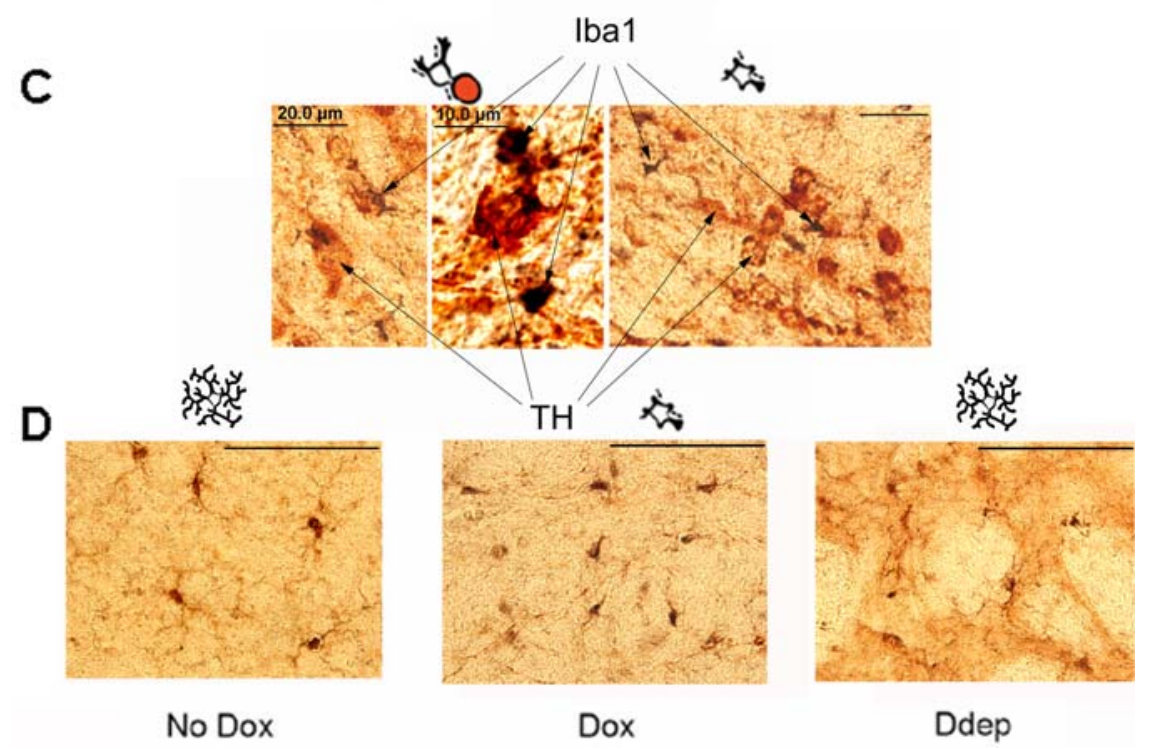

Figure 7. Global elevation in astrocytic MAO-B results in local SN microglial activation. A. Mitosox staining of local SN microglia following dox treatment reveals increased mitochondrial superoxide levels within these cells. Microglia were immunostained with lba1 (green) antibody in sections of brain fixed $3 \mathrm{hrs}$ following tail injection of mitosox red. Confocal detail shows mitochondrial mitosox red fluorescence within an activated microglial cell body following dox induction. B. Microglial activation occurs following dox induction within the SN. Microglial activation as assessed by lba1 immunochemistry (black, DAB-nickel staining) in SN sections from uninduced (No Dox), induced (Dox), and dox-induced co-deprenyl-treated transgenics (Ddep). Dopaminergic $\left(\mathrm{TH}^{+}\right)$neurons are labeled in brown (DAB). Note that few activated microglia are visible in No Dox and Ddep treated mice versus in the Dox panel where several stages of microglial activation (resting, activated, withdrawing, and motile stages) can be visualized. Scale bar equals $50 \mu \mathrm{M}$ unless indicated otherwise. C. Higher magnification of representative SN Iba1 immunochemistry demonstrating stages of microglial activation (activated, withdrawing, and motile stages are indicated by cartoons). Cortical microglia were found to be in a ramified or resting stage in all the conditions (supplementary figure S1). D. Representative lba1 immunochemistry in ST sections demonstrating increased microglial activation following dox induction. Shown are ST sections from uninduced (No Dox) versus dox-induced (Dox) or dox-induced, co-deprenyl treated (Ddep) transgenics. Note the presence of numerous retracted processes in the microglia in the Dox panel versus presence of ramified microglia in the No Dox and Ddep panels. doi:10.1371/journal.pone.0001616.g007 
A

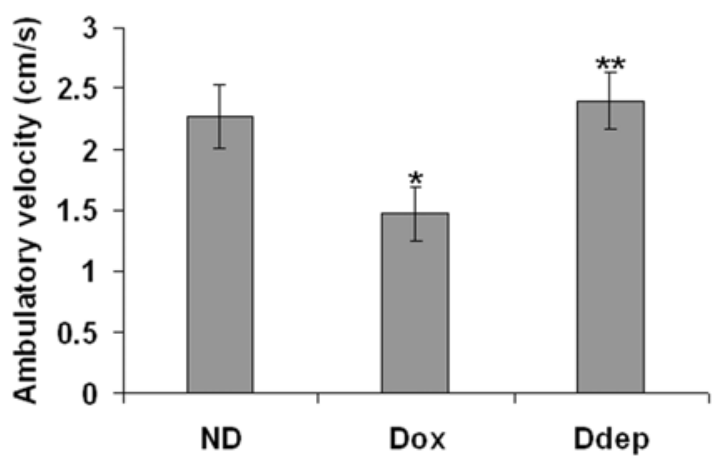

B

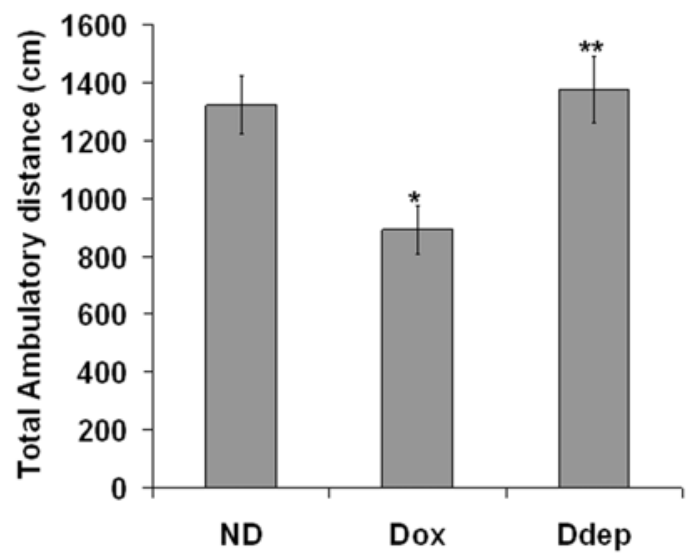

Figure 8. Elevation in astrocytic MAO-B results in loss of ambulatory movement in MAO-B transgenics. Total ambulatory velocity (A) and ambulatory distance (B) following two weeks of dox administration. Data is presented as average ambulatory velocity calculated over a 10 minute observation period and total ambulatory distance is for that same time interval. $\mathrm{N}=5$ mice per condition; experiments were run in triplicate. ${ }^{*} \mathrm{p}<0.01$ compared to ND, ** $p>0.05$ compared to ND. Values are given for untreated (ND), doxtreated (Dox), and dox, deprenyl co-treated mice. Wildtype untreated littermates had an ambulatory velocity of $2.37 \pm 0.45 \mathrm{~cm} / \mathrm{s}$ and a total ambulatory distance moved of $1082 \pm 101 \mathrm{~cm}$.

doi:10.1371/journal.pone.0001616.g008

produced by co-injecting the two transgenes into the male pronuclei of a fertilized egg prior to implantation into a pseudopregnant female. Subsequent founder mice were crossed with wildtype C57B16 and resulting litters genotyped by PCR using the primers 5'ACTGAACGCAAAGGCACAC3' and 5'AACATGACAATGAAGGAGGTAC3' for MAO-B, and 5'GCGTTTACGCATTACC3' and 5'CGCGCTTATTTTTAATGC3' for rTta. Littermates positive for both transgenes were mated to achieve homogeneous lines. Astroglial-specific transgene expression was induced by feeding animals doxycycline at 3000 ppm provided in pre-mixed Purina chow (Research Diets) for a three week period. Assuming a $5 \mathrm{~g}$ diet per day for a $30 \mathrm{~g}$ mouse, the dosage achieved was calculated to be equivalent to $0.5 \mathrm{~g} / \mathrm{kg} /$ day. Total RNA was isolated from cortical brain tissue of wildtype uninduced and uninduced transgenic mice using Trizol (Invitrogen).according to manufacturer's instructions. cDNA was prepared using Superscript III reverse transcriptase kit (Invitrogen) and oligo-(dT) primers from $2 \mu \mathrm{g}$ of total RNA. PCR was carried out with the forward and reverse primers corresponding to human MAO-B cDNA as above using Taq polymerase (Eppendorf). A 448bp amplicon indicates the induction of the h-MAO-B transcript. Positive transgene induction was also confirmed based on MAO-B and betagalactosidase positiveimmunostaining within astrocytes of dox-induced transgenics using antibodies specific to hMAO-B and betagalactosidase within $\mathrm{GFAP}^{+}$cells. Betagalactosidase and MAO-B enzyme activities were also performed in whole brain homogenates from induced versus uninduced transgenic lines [2,55]. The line showing the highest level of induction was used for all subsequent studies. Mice were housed according to standard animal care protocols, fed ad libitum, kept on a $12 \mathrm{hr}$ light/dark cycle, and maintained in a pathogen-free environment in the Buck Institute Vivarium. Animals used for subsequent studies were either young adults (2-6 months of age) or older animals (14 months of age) fed dox on the regime described above. Co-treatment with other agents involved intraperitoneal administrations of $10-30 \mathrm{mg} / \mathrm{kg} /$ day of deprenyl, minocycline or EUK-189 for two weeks.

\section{$\mathrm{MPP}^{+}$measurements, striatal dopamine content cell counts and neurodegeneration in inducible astrocytic MAO-B transgenics versus controls +/- MPTP}

Striatal tissue was harvested from dox versus untreated animals 90 minutes following injection of $30 \mathrm{mg} / \mathrm{kg}$ MPTP intraperitoneally, immediately immersed in ice cold $0.1 \mathrm{M}$ perchloric acid and sonicated. $\mathrm{MPP}^{+}$was measured by HPLC as previously described [56]. Dopamine levels were analyzed in striata dissected two weeks of induction or $24 \mathrm{hrs}$ following MPTP injection post induction by HPLC followed by a 464 pulsed electrochemical detection (Neurochemistry Core, Center for Molecular Neuroscience, Vanderbilt University). Stereological cell counts were performed on immunostained brain sections from brains harvested two weeks after induction using either antibody against tyrosine hydroxylase (TH, Chemicon, 1:500) followed by biotin-labeled secondary antibody and development using DAB (Vector Laboratories). $\mathrm{TH}^{+}$ cells were counted stereologically throughout the SNpc [56]. Counts for dox removal were performed on brains dissected after removal of dox from the feed for a three week period. Sections were cut at a $40 \mu \mathrm{m}$ thickness, and every 4 th section was counted using a grid of $100 \times 100 \mu \mathrm{m}$. Dissector size used was $35 \times 35 \times 12 \mu \mathrm{m}$. Neuronal numbers were assessed following $\mathrm{NeuN}^{+}$(Chemicon, 1:100) or GABA ${ }^{+}$(Chemicon, 1:1000) immunostaining and astrocytes via $\mathrm{GFAP}^{+}(\mathrm{DAKO}, 1: 500)$ and s100- $\beta$ (DAKO, 1:200) immunostaining. Cells were counted in the $\mathrm{SN}$, striata, and cortex in three independent sections per condition and ten fields per section. Neurodegeneration in the $\mathrm{TH}^{+}$cells was visualized by silver staining (FD Neurotechnologies, Ellicott City, MD) according to the manufacturer's instructions using proprietary compounds after immunostaining the sections with antibody against tyrosine hydroxylase ( $\mathrm{TH}$, Chemicon, 1:500) visualized with Vector Blue alkaline phosphatase (Vector labs). Dopaminochrome was measured from striatal samples by HPLC as per Ochs et al [39].

\section{Extracellular $\mathrm{H}_{2} \mathrm{O}_{2}$ levels and dopaminergic cell survival in mesencephalic cultures}

Primary mixed cultures were prepared from the midbrain of 14day-old mice embryos from MAO-B transgenics and WT controls. Tissue was digested in Neurobasal medium containing $30 \mathrm{U} / \mathrm{ml}$ papain and $20 \mu \mathrm{g} / \mathrm{ml}$ DNase at $37^{\circ} \mathrm{C}$ for $30 \mathrm{~min}$ and mechanically triturated. Dissociated cells were centrifuged at $500 \times \mathrm{g}$, resuspended in growth medium (Neurobasal medium supplemented with $10 \%$ FBS, $2 \mathrm{mM}$ glutamate, B25 supplement without antioxidants, 


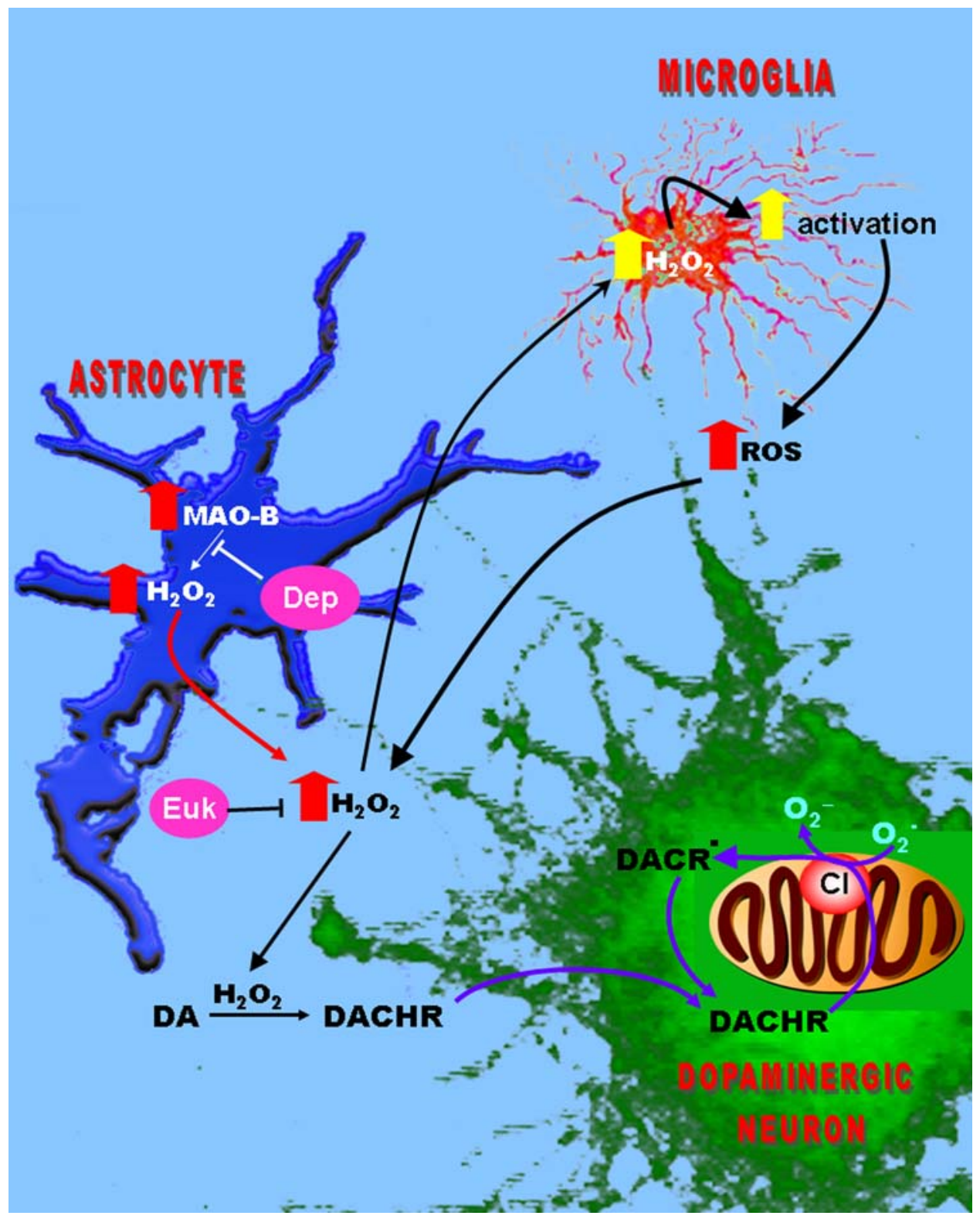

Figure 9. A schematic representation of the events occurring upon the elevation of glial MAO-B. Increased astroglial levels of MAO-B results in elevation of $\mathrm{H}_{2} \mathrm{O}_{2}$ levels; this can be prevented by deprenyl. Astroglia are themselves protected from $\mathrm{H}_{2} \mathrm{O}_{2}$ due to relatively high levels of glutathione-gluathione peroxidase versus neurons. $\mathrm{H}_{2} \mathrm{O}_{2}$ can diffuse out of astroglia and into neighboring cells including local dopaminergic neurons and microglia; this can be prevented by EUK. Dopamine can be oxidized by $\mathrm{H}_{2} \mathrm{O}_{2}$ through dopamine quinone formation to stable dopaminochrome (DACHR). DACHR can extract an electron from the auto-oxidizable site of mitochondrial complex I forming DACR radical and impacting selectively on complex I function. Due its high affinity for molecular oxygen, DACR radical can pass an electron on to this molecular species resulting in its rereduction to DACHR and increased formation of mitochondrial superoxide radical. MAO-B generated $\mathrm{H}_{2} \mathrm{O}_{2}$ can also stimulate local microglia; microglial activation can be greatly secondarily enhanced within the SN due to dopaminergic cell demise which can also exacerbate ROS production and impact on local dopaminergic neurons. doi:10.1371/journal.pone.0001616.g009

$50 \mathrm{U} / \mathrm{ml}$ penicillin, $50 \mathrm{U} / \mathrm{ml}$ streptomycin and $50 \mathrm{ng} / \mathrm{ml} \mathrm{GDNF}$ [57]), and plated on poly-d-lysine-coated 8 well chamber slides (BDBiocoat) at a density of $10^{5}$ cells per ml. Mixed cultures were grown at $37^{\circ} \mathrm{C}$ for $3-5$ days before induction with $40 \mu \mathrm{g} / \mathrm{ml}$ doxycycline for 12 hours as per [22]. Cells were co-treated with $10 \mu \mathrm{M}$ deprenyl or $500 \mathrm{nM}$ apocynin. $\mathrm{H}_{2} \mathrm{O}_{2}$ in the medium was assayed by Amplex Red per the manufacturer's instructions. Dopaminergic and microglial cell counts were performed following fixation of the cultured cells for $15 \mathrm{~min}$ with $4 \%$ formaldehyde following by $\mathrm{TH}^{+}$ (Chemicon) or $\mathrm{Ibal}^{+}$(WAKO USA, Richmond, VA) immunostain- ing or via uptake of $\mathrm{ASP}^{+}$(4-(4-(dimethylamino)styryl)- $\mathcal{N}$ - methylpyridinium iodide) (Invitrogen), a fluorescent analogue of dopamine. $10 \mu \mathrm{M} \mathrm{ASP}^{+}$was loaded on to the uninduced or induced cultures in the presence of $20 \mu \mathrm{M}$ of desipramine to inhibit the norepinephrine transporter which would also take up the dye. $\mathrm{ASP}^{+}$fluorescence (red) was visualized in the $\mathrm{TH}^{+}$cells after immunostaining with antiTH antibody and Alexa fluor 488 conjugated secondary antibody. Averages of manual double blind $\mathrm{TH}^{+}$or fluorescent counts of three wells of culture with the same treatment were collected; counts were performed in triplicate. 
Immuno-magnetic isolation of dopaminergic synaptosomes

Dopaminergic and non-dopaminergic striatal synaptosomes were isolated using a modified immuno-magnetic protocol [58]. Briefly, synaptosomes were prepared from dissected striatal tissue [59]. Dopaminergic synaptosomes were isolated using magnetic beads conjugated to anti-rabbit IgG (Miltenyi Biotech) after capturing them using a rabbit anti-DAT antibody (Chemicon). Under a magnetic field, bead-bound synaptosomes were passed through a column and washed; flow-through in the presence of the magnetic field yielded a fraction enriched in non-dopaminergic striatal synaptosomes. Elution performed in the absence of the magnetic field yielded fractions enriched in dopaminergic striatal synaptosomes. Equal quantities of various fractions of the synaptosomes isolated using DAT antibody including the starting material or control, the unbound fraction, the flow-through, the wash and the eluate were subjected to western analysis to assess relative dopaminergic versus non-dopaminergic synaptosomal purification. Western blots were performed using antibodies against TH (Chemicon 1:500), GABA (Chemicon, 1:1000) or SNAP-25 as a synaptic protein normalization control (Chemicon, 1:1000). Band densities were quantified using a Chemimager (Alpha Innotech, San Leandro, CA). Approximately 94\% synaptosomes in the eluate were dopaminergic according to densitometric evaluation of the western blot.

\section{$\mathrm{H}_{2} \mathrm{O}_{2}$ estimation in synaptosomes}

Hydrogen peroxide levels were measured in the striatal dopaminergic and non-dopaminergic synaptosomes prepared as above from various groups of mice. Mice were injected in the tail vein with $\sim 200 \mu \mathrm{l}$ of DCFDA (Calbiochem) which was freshly diluted $100 \mathrm{x}$ with PBS from a stock of $100 \mathrm{mg} / \mathrm{ml}$ in DMSO as modified from Andrews et. al. [38]. The animals were sacrificed 3 hours later and synaptosomes were prepared as above. Hydrogen peroxide in the synaptosomes with or without $1 \mathrm{mg}$ / $\mathrm{ml}$ catalase for $30 \mathrm{~min}$ after preparation was visualized as DCF fluorescence at $488 \mathrm{~nm}$ excitation and $512 \mathrm{~nm}$ emission in a Spectramax Gemini fluorescence plate reader. The relative fluorescence was normalized to synaptosomal protein which was quantified using the Bradford reagent, Bio-Rad.

\section{Mitochondrial complex I and IV activities}

Isolated synaptosomes were found to be physiologically viable for up to 3 hours. Complex I activities were assayed in isolated dopaminergic and non-dopaminergic synaptosomal fractions from dox-induced animals as rotenone-sensitive NADH dehydrogenase activity by measuring DCPIP (2,6-dichlorophenolindophenol) reduction in synaptosomal extracts following addition of $200 \mu \mathrm{M}$

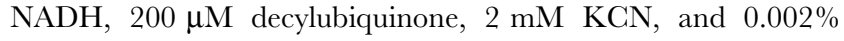
DCPIP in the presence and absence of $2 \mu \mathrm{M}$ rotenone [60]. Complex IV activity was assayed as cytochrome c oxidase activity by observing the rapid (1-2s) oxidation of freshly reduced $40 \mu \mathrm{M}$ ferrocytochrome $\mathrm{c}$ in a $10 \mathrm{mM} \mathrm{K}-\mathrm{PO}_{4}$ buffer $\mathrm{pH} 7.2$ containing $100 \mathrm{mM} \mathrm{KCl}, 0.025 \%$ maltoside at $30^{\circ} \mathrm{C}$, at $550 \mathrm{~nm}$, averaged over two concentrations per sample. Values for all assays were normalized/protein using BioRad reagent.

\section{Detection of mitochondrial superoxide in vivo}

Mitosox Red (Molecular Probes) was used as an indicator to assess presence of superoxide within cellular mitochondria. $10 \mu \mathrm{g}$ of Mitosox in $200 \mu \mathrm{l}$ phosphate-buffered saline was injected into the tail vein of dox-induced transgenics versus controls. Animals were sacrificed 90 minutes later, brains rapidly dissected out and post-fixed overnight in $4 \%$ paraformaldehyde. Brains were sectioned at $30 \mu \mathrm{m}$ using a cryostat. Cellular localization and site of Mitosox Red accumulation in the nigrostriatum was identified by immunofluorescence using anti-TH (Chemicon, 1:500), antiIBA1 (Wako, 1:500) or anti-GFAP (DAKO, 1:500) antibodies visualized with Fluorescein Avidin D. Mitochondrial localization was confirmed by immunoanalysis using an anti-ATP synthasespecific antibody. Fluorescent imaging was done using a Zeiss LSM510 NLO confocal microscope and images procured using LSM software. Quantification of colocalization and fluorescent intensity was determined using the IMARIS software suite (Bitplane AG, Zurich, Switzerland) on selected cells from multiple sections.

\section{Morphological analyses of astrocyte and microglial activation}

Immunochemistry was performed on cryosections derived from the SN, striata, and CTX of fixed perfused brains from doxinduced transgenics versus controls. Astrocytic activation was assessed using primary antibodies against GFAP and s100- $\beta$. Microglial activation was detected using primary antibodies against Ibal. Horse radish peroxidase or avidin-conjugated secondary antibodies were used to detect primary antibodies following either DAB or Fluorescein/Texas Red staining.

\section{Locomotor behavioral assessment}

Locomotor function was assessed using an automated Truscan photobeam apparatus (Colbourne Instruments, Allentown, PA) under illumination as previously described [61]. Animals were habituated to the apparatus for $10 \mathrm{~min}$ then data collected over a 10-min period followed by analysis using Truscan 99 software. Spontaneous horizontal movement was assessed in both control and dox treated versus untreated transgenic mice via both ambulatory velocity and distance in a 10 -min period.

\section{Supporting Information}

Figure S1 Microglial activation is largely absent in the cortex. Absence of detectable microglial activation in the cortical sections from untreated, dox-treated deprenyl co-treated animals. Ibal immunocytochemistry reveals that cortical microglia are in a resting ramified stage in all the three types of treatments conditions in contrast to what occurs in the striatum and the substantia nigra (Figure 6). This suggests that activation in the nigrostriatum may largely be due to secondary effects as a consequence of SN dopaminergic demise. Two representative micrographs are provided from each treatment.

Found at: doi:10.1371/journal.pone.0001616.s001 (10.35 MB TIF)

\section{Acknowledgments}

We thank Raymond Johnson (Vanderbilt University, Nashville Tennessee) for measurement of dopamine content and Martha Isla (Parkinson's Institute, Sunnyvale, CA) for $\mathrm{MPP}^{+}$measurements.

\section{Author Contributions}

Conceived and designed the experiments: DN JM JA. Performed the experiments: JM DK SG AR DD HM SR. Analyzed the data: DN JM JA DK SC. Contributed reagents/materials/analysis tools: DN JM DK SC AR DD HM SR. Wrote the paper: JM JA. 


\section{References}

1. Westlund KN, Denney RM, Rose RM, Abell CW (1988) Localization of distinct monoamine oxidase A and monoamine oxidase B cell populations in human brainstem. Neuroscience 25: 439-456.

2. Westlund KN, Denney RM, Kochersperger LM, Rose RM, Abell CW (1985) Distinct monoamine oxidase A and B populations in primate brain. Science 230: 181-183.

3. Levitt P, Pintar JE, Breakefield XO (1982) Immunocytochemical demonstration of monoamine oxidase B in brain astrocytes and serotonergic neurons. Proc Natl Acad Sci U S A 79: 6385-6389.

4. Saura J, Richards JG, Mahy N (1994) Age-related changes on MAO in Bl/C57 mouse tissues: a quantitative radioautographic study. J Neural Transm Suppl 41: 89-94.

5. Fowler CJ, Wiberg A, Oreland L, Marcusson J, Winblad B (1980) The effect of age on the activity and molecular properties of human brain monoamine oxidase. J Neural Transm 49: 1-20.

6. Riederer P, Konradi C, Schay V, Kienzl E, Birkmayer G, et al. (1987) Localization of MAO-A and MAO-B in human brain: a step in understanding the therapeutic action of L-deprenyl. Adv Neurol 45: 111-118.

7. Gerlach M, Youdim MB, Riederer P (1996) Pharmacology of selegiline. Neurology 47: S137-145.

8. Vindis C, Seguelas MH, Lanier S, Parini A, Cambon C (2001) Dopamine induces ERK activation in renal epithelial cells through $\mathrm{H} 2 \mathrm{O} 2$ produced by monoamine oxidase. Kidney Int 59: 76-86.

9. Cohen G, Farooqui R, Kesler N (1997) Parkinson disease: a new link between monoamine oxidase and mitochondrial electron flow. Proc Natl Acad Sci U S A 94: 4890-4894.

10. Adams JD Jr, Odunze IN (1991) Oxygen free radicals and Parkinson's disease. Free Radic Biol Med 10: 161-169.

11. Halliwell B (1992) Reactive oxygen species and the central nervous system. J Neurochem 59: 1609-1623.

12. Raps SP, Lai JC, Hertz L, Cooper AJ (1989) Glutathione is present in high concentrations in cultured astrocytes but not in cultured neurons. Brain Res 493: 398-401.

13. Sagara JI, Miura K, Bannai S (1993) Maintenance of neuronal glutathione by glial cells. J Neurochem 61: 1672-1676.

14. Makar TK, Nedergaard M, Preuss A, Gelbard AS, Perumal AS, et al. (1994) Vitamin E, ascorbate, glutathione, glutathione disulfide, and enzymes of glutathione metabolism in cultures of chick astrocytes and neurons: evidence that astrocytes play an important role in antioxidative processes in the brain. J Neurochem 62: 45-53.

15. Kang Y, Oiao X, Jurma O, Knusel B, Andersen JK (1997) Cloning/brain localization of mouse glutamylcysteine synthetase heavy chain mRNA. Neuroreport 8: 2053-2060.

16. Buckman TD, Sutphin MS, Mitrovic B (1993) Oxidative stress in a clonal cell line of neuronal origin: effects of antioxidant enzyme modulation. J Neurochem 60: 2046-2058.

17. Behl C, Davis JB, Lesley R, Schubert D (1994) Hydrogen peroxide mediates amyloid beta protein toxicity. Cell 77: 817-827.

18. Whittemore ER, Loo DT, Cotman CW (1994) Exposure to hydrogen peroxide induces cell death via apoptosis in cultured rat cortical neurons. Neuroreport 5 : 1485-1488.

19. Wei Q Yeung M, Jurma OP, Andersen JK (1996) Genetic elevation of monoamine oxidase levels in dopaminergic PC12 cells results in increased free radical damage and sensitivity to MPTP. J Neurosci Res 46: 666-673.

20. Damier P, Kastner A, Agid Y, Hirsch EC (1996) Does monoamine oxidase type B play a role in dopaminergic nerve cell death in Parkinson's disease? Neurology 46: $1262-1269$.

21. Soong NW, Hinton DR, Cortopassi G, Arnheim N (1992) Mosaicism for a specific somatic mitochondrial DNA mutation in adult human brain. Nat Genet 2: 318-323.

22. Kumar MJ, Nicholls DG, Andersen JK (2003) Oxidative alpha-ketoglutarate dehydrogenase inhibition via subtle elevations in monoamine oxidase B levels results in loss of spare respiratory capacity: implications for Parkinson's disease. J Biol Chem 278: 46432-46439.

23. Betarbet R, Sherer TB, MacKenzie G, Garcia-Osuna M, Panov AV, et al. (2000) Chronic systemic pesticide exposure reproduces features of Parkinson's disease. Nat Neurosci 3: 1301-1306.

24. Przedborski S, Vila M (2003) The 1-methyl-4-phenyl-1,2,3,6-tetrahydropyridine mouse model: a tool to explore the pathogenesis of Parkinson's disease. Ann N Y Acad Sci 991: 189-198.

25. Gainetdinov RR, Fumagalli F, Jones SR, Caron MG (1997) Dopamine transporter is required for in vivo MPTP neurotoxicity: evidence from mice lacking the transporter. J Neurochem 69: 1322-1325.

26. Wu RM, Murphy DL, Chiueh CC (1996) Suppression of hydroxyl radical formation and protection of nigral neurons by 1-deprenyl (selegiline). Ann N Y Acad Sci 786: 379-390.

27. Magyar K, Szende B (2004) (-)-Deprenyl, a selective MAO-B inhibitor, with apoptotic and anti-apoptotic properties. Neurotoxicology 25: 233-242.

28. Szende B, Bokonyi G, Bocsi J, Keri G, Timar F, et al. (2001) Anti-apoptotic and apoptotic action of (-)-deprenyl and its metabolites. J Neural Transm 108: 25-33.
29. Shoulson I (1992) An interim report of the effect of selegiline (L-deprenyl) on the progression of disability in early Parkinson's disease. The Parkinson Study Group. Eur Neurol 32 Suppl 1: 46-53.

30. LeWitt PA (1994) Clinical trials of neuroprotection in Parkinson's disease: longterm selegiline and alpha-tocopherol treatment. J Neural Transm Suppl 43: $171-181$.

31. Schneider E (1995) DATATOP-study: significance of its results in the treatment of Parkinson's disease. J Neural Transm Suppl 46: 391-397.

32. Parkinson_Study_Group (1998) Mortality in DATATOP: a multicenter trial in early Parkinson's disease. Parkinson Study Group. Ann Neurol 43: 318-325.

33. Fearnley JM, Lees AJ (1991) Ageing and Parkinson's disease: substantia nigra regional selectivity. Brain 114 ( Pt 5): 2283-2301.

34. Zoccarato F, Toscano P, Alexandre A (2005) Dopamine-derived dopaminochrome promotes $\mathrm{H}(2) \mathrm{O}(2)$ release at mitochondrial complex I: stimulation by rotenone, control by $\mathrm{Ca}(2+)$, and relevance to Parkinson disease. J Biol Chem 280: 15587-15594.

35. Pickel VM, Johnson E, Carson M, Chan J (1992) Ultrastructure of spared dopamine terminals in caudate-putamen nuclei of adult rats neonatally treated with intranigral 6-hydroxydopamine. Brain Res Dev Brain Res 70: 75-86.

36. Nirenberg MJ, Chan J, Liu Y, Edwards RH, Pickel VM (1996) Ultrastructural localization of the vesicular monoamine transporter-2 in midbrain dopaminergic neurons: potential sites for somatodendritic storage and release of dopamine. J Neurosci 16: 4135-4145.

37. Nirenberg MJ, Vaughan RA, Uhl GR, Kuhar MJ, Pickel VM (1996) The dopamine transporter is localized to dendritic and axonal plasma membranes of nigrostriatal dopaminergic neurons. J Neurosci 16: 436-447.

38. Andrews ZB, Horvath B, Barnstable CJ, Elsworth J, Yang L, et al. (2005) Uncoupling protein-2 is critical for nigral dopamine cell survival in a mouse model of Parkinson's disease. J Neurosci 25: 184-191.

39. Ochs SD, Westfall TC, Macarthur H (2005) The separation and quantification of aminochromes using high-pressure liquid chromatography with electrochemical detection. J Neurosci Methods 142: 201-208.

40. McGeer PL, Itagaki S, Boyes BE, McGeer EG (1988) Reactive microglia are positive for HLA-DR in the substantia nigra of Parkinson's and Alzheimer's disease brains. Neurology 38: 1285-1291.

41. Langston JW, Forno LS, Tetrud J, Reeves AG, Kaplan JA, et al. (1999) Evidence of active nerve cell degeneration in the substantia nigra of humans years after 1methyl-4-phenyl-1,2,3,6-tetrahydropyridine exposure. Ann Neurol 46: 598-605.

42. McGeer PL, Schwab C, Parent A, Doudet D (2003) Presence of reactive microglia in monkey substantia nigra years after 1-methyl-4-phenyl-1,2,3,6tetrahydropyridine administration. Ann Neurol 54: 599-604.

43. Sherer TB, Betarbet R, Testa CM, Seo BB, Richardson JR, et al. (2003) Mechanism of toxicity in rotenone models of Parkinson's disease. J Neurosci 23: 10756-10764.

44. Peng J, Xie L, Stevenson FF, Melov S, Di Monte DA, et al. (2006) Nigrostriatal dopaminergic neurodegeneration in the weaver mouse is mediated via neuroinflammation and alleviated by minocycline administration. J Neurosci 26: 11644-11651.

45. Imai Y, Kohsaka S (2002) Intracellular signaling in M-CSF-induced microglia activation: role of Iba1. Glia 40: 164-174.

46. Stence N, Waite M, Dailey ME (2001) Dynamics of microglial activation: a confocal time-lapse analysis in hippocampal slices. Glia 33: 256-266.

47. Youdim MB, Bakhle YS (2006) Monoamine oxidase: isoforms and inhibitors in Parkinson's disease and depressive illness. Br J Pharmacol 147 Suppl 1: S287-296.

48. Mandel S, Grunblatt E, Riederer P, Gerlach M, Levites Y, et al. (2003) Neuroprotective strategies in Parkinson's disease : an update on progress. CNS Drugs 17: 729-762.

49. Irwin I, Finnegan KT, Delanney LE, Di Monte D, Langston JW (1992) The relationships between aging, monoamine oxidase, striatal dopamine and the effects of MPTP in C57BL/6 mice: a critical reassessment. Brain Res 572: $224-231$.

50. Cohen G (1983) The pathobiology of Parkinson's disease: biochemical aspects of dopamine neuron senescence. J Neural Transm Suppl 19: 89-103.

51. Olanow CW (1993) A rationale for monoamine oxidase inhibition as neuroprotective therapy for Parkinson's disease. Mov Disord 8 Suppl 1: S1-7.

52. Cicchetti F, Brownell AL, Williams K, Chen YI, Livni E, et al. (2002) Neuroinflammation of the nigrostriatal pathway during progressive 6-OHDA dopamine degeneration in rats monitored by immunohistochemistry and PET imaging. Eur J Neurosci 15: 991-998.

53. Schneider JS, Denaro FJ (1988) Astrocytic responses to the dopaminergic neurotoxin 1-methyl-4-phenyl-1,2,3,6-tetrahydropyridine (MPTP) in cat and mouse brain. J Neuropathol Exp Neurol 47: 452-458.

54. Sugama S, Yang L, Cho BP, DeGiorgio LA, Lorenzl S, et al. (2003) Age-related microglial activation in 1-methyl-4-phenyl-1,2,3,6-tetrahydropyridine (MPTP)induced dopaminergic neurodegeneration in C57BL/6 mice. Brain Res 964: 288-294.

55. Miller JH (1972) Experiments in Molecular Genetics, Cold Spring Harbor, N.Y.: Cold Spring Harbor Laboratory Press.

56. Kaur D, Yantiri F, Rajagopalan S, Kumar J, Mo JQ et al. (2003) Genetic or pharmacological iron chelation prevents MPTP-induced neurotoxicity in vivo: a novel therapy for Parkinson's disease. Neuron 37: 899-909. 
57. Smith DW, Friedmann T (2004) Discrepant effects of culture conditions on survival and function of dopaminergic neurons. Neuroreport 15: 1025-1028.

58. Docherty M, Bradford HF, Cash CD, Ehret M, Maitre M, et al. (1991) Isolation of monoaminergic synaptosomes from rat brain by immunomagnetophoresis. J Neurochem 56: 1569-1580.

59. Budd DC, Nicholls DG (1995) Protein kinase C-mediated suppression of the presynaptic adenosine A1 receptor by a facilitatory metabotropic glutamate receptor. J Neurochem 65: 615-621.
60. Trounce IA, Kim YL, Jun AS, Wallace DC (1996) Assessment of mitochondrial oxidative phosphorylation in patient muscle biopsies, lymphoblasts, and transmitochondrial cell lines. Methods Enzymol 264: 484-509.

61. Peng J, Wu Z, Wu Y, Hsu M, Stevenson FF, et al. (2002) Inhibition of caspases protects cerebellar granule cells of the weaver mouse from apoptosis and improves behavioral phenotype. J Biol Chem 277: 44285-44291.

62. Yadava N, Houchens T, Potluri P, Scheffler IE (2004) Development and characterization of a conditional mitochondrial complex I assembly system.J Biol Chem 279: 12406-12413. 